\title{
Wie schützen wir unsere Angen vor der Einwirkung der nltravioletten Strahlen unserer künstlichen Lichtquellen?
}

Von

Augenarzt San.-Rat Dr. Fritz Schanz u.Dr.-Ing. Karl S tockhausen, beide in Dresden.

Mit Taf. V-XIV.

$\mathrm{Zu}$ der vorliegenden Untersuchung gab ein Leiden eines der Autoren den Anlass.

Herr Dr.-Ing. Stockhausen hatte bei Untersuchungen über den elektrischen Lichtbogen eine halbe Stunde lang denselben fast ununterbrochen mit dem Auge beobachtet. Obgleich er bei dieser Untersuchung seine gewöhnliche helle Glasbrille trug, und die Lampe mit einer Glasglocke umgeben war, erkrankte er trotzdem an einer heftigen Augenentzündung. Die ersten Beschwerden stellten sich nach einigen Stunden ein. Er fühlte Schmerzen und eine Abnahme der Sehkraft. Die Schmerzen steigerten sich derart, dass ihm das Lesen einer Zeitung unmöglich wurde. Sie wurden immer heftiger, zu den stechenden Schmerzen kam ein reibendes Gefühl und starke Tränenabsonderung. Dabei waren die Augen stark gerötet und verschwollen. In der Nacht war das Schmerzgefühl so intensiv, dass an ein Schlafen nicht $z u$ denken war. Am Morgen zeigten sich die Augen stark mit dem abgesonderten Sekret verklebt. Das Öffnen liess sich nur unter grossen Schmerzen ermöglichen. Die Sehschärfe war sehr gering, und es bestand grosse lichtscheu. Als der Patient am nächsten Tage untersucht wurde, fand sich neben den äusserlich sichtbaren Erscheinungen einer Ophthalmia electrica auch ein kleiner Verbrennungsfleck in der Netzhautmitte. Auch heute besteht davon noch ein kleines rechteckiges Skotom, das neben dem Fixationspunkt liegt.

Das auffälligste an dem Fall war, dass die elektrische Ophthalmie aufgetreten war, trotzdem der Patient eine Brille trug und die

v. Graefe's Archiv für Ophthalmologie. LXIX. 1. 
Lampe mit einer Glocke umgeben war. Die Ophthalmia electrica gilt nämlich als eine Wirkung der ultravioletten Strahlen und nach den Untersuchungen von Widmark, Hertel, Birch-Hirschfeld sollte das Vorschalten einer Glasplatte schon genügen, sämtliche Erscheinungen abzuhalten, die durch intensives ultraviolettes Licht hervorgerufen werden. In dem eingangs angeführten Fall hatten die Lampenglocke und die Brillengläser nicht ausgereicht, um die schädliche Wirkung dieser Strahlen auf das Auge zu verhindern.

Diese Erkenntnis gab uns den Anlass die Frage aufzustellen: Wie schützen wir unsere Augen vor den schädlichen Wirkungen der ultravioletten Strahlen unserer künstlichen Iichtquellen?

Unsere Untersuchungen zur Beantwortung dieser Frage lassen sich in vier Abschnitte zergliedern:

I. Der Reichtum unserer künstlichen Lichtquellen an ultravioletten Strahlen.

II. Der schädliche Einfluss der ultravioletten Strahlen auf das Auge.

III. Die Absorptionsfähigkeit der Glassorten gegenüber ultravioletten Strahlen.

IV. Der Schutz des Auges gegen ultraviolette Strahlen.

\section{Der Reichtum unserer künstlichen Lichtquellen an ultravioletten} Strahlen.

Bei der Einwirkung des Lichtes auf das Auge müssen wir zwischen der Wirkung der sichtbaren und der unsichtbaren Strahlen unterscheiden. Beide werden häufig nicht genügend auseinander gehalten. Die ersteren führen, wenn sie in zu grosser Lichtstärke auf das Auge einwirken, zur Blendung, die zweiten, die unsichtbaren Strahlen erzeugen am Auge Reizerscheinungen, wie wir sie am stärksten ausgeprägt bei der Ophthalmia electrica finden. Ähnliche Entzündungserscheinungen können durch jede Lichtquelle, die genügend reich an ultravioletten Strahlen ist, hervorgerufen werden. Unsere Untersuchungen haben uns gezeigt, dass neben dem elektrischen Licht auch andere Lichtquellen eine beträchtliche Menge dieser Strahlen besitzen. Die heftigsten Augenentzündungen dieser Art sind an Personen beobachtet worden, die am elektrischen Lichtbogen beschäftigt waren. Bei diesen Kranken hat man zuerst erkannt, dass diese Veränderungen ein charakteristisches Krankheitsbild darstellen, daher der Name. - 
Den Schutz des Auges gegen Blendung wollen wir in dieser Abhandlung ganz ausschalten, da sie sonst einen zu grossen Umfang annehmen würde.

Schon seit den ersten Anfängen der Beleuchtungstechnik macht sich das Bestreben geltend, die Temperatur der Lichtquellen immer mehr zu steigern, einmal um grőssere Lichtstärken zu erzielen, zum andern das Licht im Preise billiger zu gestalten. Als das eine der Ziele der Leuchttechnik gilt: ideale Leuchtkörper zu suchen, welche auf die grösstmögliche Temperatur erhitzt werden können und in ihr feuerbeständig sind ${ }^{1}$. Nun ist bekannt, dass mit steigender Temperatur die Energie jeder Wellenlänge wächst und dabei die Energievermehrung um so grösser wird, je kleiner die Wellenlänge ist. Es wandert also bei steigender Temperatur eines Körpers sein Energiemaximum immer mehr nach dem ultravioletten Ende des Spektrums. Oder mit andern Worten, je höher die Temperatur eines leuchtenden Körpers ist, desto grösser ist der Anteil der ultravioletten, nicht sichtbaren Strahlen an der Gesamtstrahlung. Da in der neuen Zeit festgestellt worden ist, dass die ultravioletten Strahlen dem menschlichen Körper und besonders auch dem Auge schädlich sind und dass sich dabei ein Unterschied zwischen der Wirkung der ultravioletten Strahlen grösserer und kürzerer Wellenlänge ergibt, lag uns daran, experimentell und auch qualitativ die Zunahme dieser Strahlen mit der Entwicklung der Leuchttechnik bis auf den heutigen Stand genauer festzustellen und die verschiedensten Lichtquellen darauf zu vergleichen.

Im hygienischen Institut der Dresdener Technischen Hochschule fanden wir dazu Gelegenheit. Herr Geheimer Medizinalrat Prof. Dr. Renk, dem wir hierfür unsern verbindlichsten Dank aussprechen, stellte uns sowohl eine reiche Sammlung der verschiedensten Lampen als auch sein Laboratorium zur Verfügung.

Versuchsanordnung: Alle in den Taf. $V$-XIV wiedergegebenen Spektren wurden mit einem Spektrographen aufgenommen, dessen Linsensysteme (Quarz-Fluoritachromaten) und Cornu-Prisma aus Quarz bestanden. Der Apparat war uns von Herrn Dr. H. Thiele freundlichst für alle unsere Untersuchungen zur Verfügung gestellt und auch ihm sei an dieser Stelle nochmals unser ganz besonderer Dank ausgesprochen.

Unsere Untersuchungen erstreckten sich von der römischen Oliven-

1) O. Lummer, Die Ziele der Leuchttechnik. Verlag von Oldenburg. 1903. 
öllampe anfangend über alle charakteristischen Öllampen, Gaslampen, bis zu den elektrischen Glüh- und Bogenlampen.

Zum besseren Verständnis der Tafeln mögen noch folgende Erklärungen dienen. Die beiden ersten durchgehenden Linien auf den Tafeln kennzeichnen die Ausdehnung des sichtbaren Spektrums, von Wellenlängen $770 \mu \mu$ bis $390 \mu \mu$. Die erste Linie zeigt den Anfang des Spektrums im Rot. Die zweite bildet die Grenze zwischen den sichtbaren und den unsichtbaren Strahlen. Die dritte Linie stellt die mittlere Absorptionsgrenze für gewöhnliches Glas dar. Links von den Spektren sind die jemaligen Beleuchtungszeiten angegeben. Die Erklärungen auf der rechten Seite geben über die Lichtquellen, deren Lichtstärken, die Glassorten und Glasdicken Auskunft, von denen das betreffende Spektrum herrührt.

1. Öllampen. Im grauen Altertum diente das Herdfeuer auch als Lichtquelle. Ein rom Herde gerissener Span, mit dem dem scheidenden Gaste ein Stück seines Weges erleuchtet wurde, kennzeichnete die erste Trennung des Lichtes vom wärmenden Feuer, die in der Folge zur Herstellung von besonderen Fackeln aus Holz in Verbindung mit Harz und Pech oder aus Kienholz führte. Die Umhüllung von Binsen mit Bienenwachs gab den ersten Anstoss zur Herstellung von Kerzen, wie sie uns heute dank dem mächtigen Aufblühen der chemischen Technik in den verschiedensten Arten zur Verfügung stehen. Den nahe liegenden Gedanken, an Stelle festen Brennmaterials und fester Fettstoffe flüssige Fette und Öle in flachen. Schalen, aus denen ein Faserdocht hervorragte, zu verwenden, finden wir schon bei den alten Assyrem und Ägyptern verwirklicht. In der Form der römischen Olivenöllampen hat sich die Antikenlampe durch viele Jahrhunderte hindurch fast unverändert bis in das 18. Jahrhundert erhalten. Den ersten wesentlichen Aufschwung erfuhr die Beleuchtungstechnik durch die Einführung des Cylinders, Zugglases, wie er am Anfang bezeichnet wurde, durch den Apotheker Quinquet (1765). Ihm folgte der Hohldocht, eine Erfindung des Grafen Argand (1786), der sich auch auf dem Gebiete der Gasbeleuchtang durch seinen Argandbrenner einen Namen geschaffen hat. Diese den ersten Anschein nach geringfügigen Erfindungen waren doch von einschneidender Wichtigkeit, denn sie allein gestatteten zum ersten Male eine rationelle Verwendung des Beleuchtungsmaterials und schufen anstatt der qualmenden Öllampe, wenn auch kein besonders helles, so doch ein ruhig brennendes Licht. Bis zum Jahre 1859 blieb die Rüböllampe unbestrittene Herrscherin. In 
diesem Jahre wurde das Petroleum von Nordamerika nach Europa eingeführt und in dem Wettkampf mit der Petroleumlampe, die selbst dem damals schon ziemlich ausgebreiteten Leuchtgas Konkurrenz machte, unterlag die Rüböllampe bald. Die Petroleumlampe aber wurde zum wichtigsten Beleuchtungsapparat; sie selbst hat heute noch neben den modernsten elektrischen Glühlampen und Gasglühlampen nicht an Bedeutung eingebïsst. Auch an Verbesserungen der Petroleumlampe hat es nicht gefehlt. Die Versuche, die heisse, nicht leuchtende Spiritusflamme durch Einbringen von Glühkörpern als Lichtquelle zu verwenden, und welche die Grundlage zur Erfindung der Auer-Strümpfe bilden, führten schliesslich zur Konstruktion von Petroleum und Spiritus-Glühlampen. Das Problem wirklich brauchbarer Konstruktionen wurde aber erst in den letzten Jahren gelöst.

Welchen Einfluss hat diese Entwicklung der Öllampen nun auf das Spektrum der einzelnen Lichtquellen ausgeübt? Aus den Strahlungsgesetzen können wir nur wieder die Behauptung aufstellen: die Lichtquellen, die eine höhere Temperatur wie andere besitzen, müssen auch ein ausgedehnteres Spektrum haben. Sofort wird sich hieraus eine zweite Frage ergeben: In welcher Reihenfolge sind die Öllampen ihrer Temperatur nach zu ordnen? Zur Beantwortung dieser Frage müssen wir uns klar machen, durch welche Umstände eine Erhöhung der Temperatur der Lichtquellen bewerkstelligt wird. Einmal kann dies der Fall sein durch eine leichtere und schnellere Verdampfung der brennbaren Fette und Öle. Die zweite Möglichkeit gewährt eine vermehrte Sauerstoffzufuhr, wodurch die Verbrennung eine intensivere wird, jedoch darf die Luftzufuhr nicht so gross sein, dass die glühenden Kohlenteilchen sofort verbrennen, ohne erst eine gewisse Zeit lang in Weissglut erhalten zu bleiben. Einen dritten Weg hat uns Auer von Welsbach gewiesen. In einer entleuchteten und darum sehr heissen Flamme wird ein Körper von grosser Leuchtfähigkeit gebracht, der nun, durch die sehr heisse Flamme auf eine hohe Temperatur erhitzt, selbst anfängt, Lichtstrahlen von grosser Stärke auszusenden.

Betrachten wir unter diesen Gesichtspunkten die Spektren auf Taf. V. Die Spektren 1-4 römische-Olivenöllampe, Walrat- und Stearinkerzen, offene Rüböllampe, unterscheiden sich in ihrer Ausdehnung kaum voneinander. Thr Spektrum reicht wohl bis in das Ultraviolett, aber man kann sie als arm an solchen Strahlen bezeichnen.

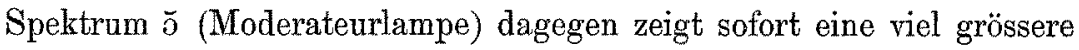
Ausdehnung, obgleich auch nur Rüböl als Brennstoff dient. Es ist 
dies allein darauf zurückzufühiren, dass durch den Cylinder ein starker Zug hervorgerufen und dadurch mehr Luft zur besseren Verbrennung und zur Erhöhung der Temperatur hinzugeführt wird. Dasselbe gilt für die Petroleum-Schnitt- und Rundbrenner, Spektrum 7 und 8, sowie für Mays Ideallampe, Spektrum 6. Bei der letzteren übernimmt nur ein stetig wirkendes und durch ein Uhrwerk getriebenes Gebläse die Stelle des Cylinders. Die Lampen mit Cylinder nehmen also schon einen grösseren Anteil an der ultravioletten Strahlung als die Kerzen und offenen. Rüböllampen, können aber immerhin im Vergleich zu unseren modernen Lichtquellen, wie wir später zeigen werden, als relativ arm an ultravioletten Strahlen bezeichnet werden. Eine bedeutende Länge und einen grossen Reichtum an ultravioletten Strahlen lassen aber die beiden Spektren 9 und 10, von einer Petroleum- und Spiritusglühlampe, erkennen, die von diesen Lampen auch die höchste Temperatur besitzen.

2. Gaslampen. Auf dieselbe Weise wie bei den Öllampen lässt sich auch bei den Gaslampen, Taf. VI, das Anwachsen der ultravioletten Strahlung bei Erhöhung der Temperatur der Flammen beobachten. Sein erster Darsteller Becher (1685), der es in seiner überschwenglichen Freude „philosophisches Licht" nannte, ahnte wohl kaum, dass es eine so allgemeine Verbreitung finden würde, wie das heutzutage der Fall ist. In der Tat führte es sich auch anfangs sehr schwer ein, erst im Jahre 1812 erhielt London seine erste Gasbeleuchtung, Berlin șogar erst im Jahre 1826. Da das Gas, wie bei allen früheren Beleuchtungsarten, hauptsä̈chlich aus Kohlenstoff und Wasserstoff besteht, so müssen die Brenner so eingerichtet sein, dass eine möglichst heisse Flamme entsteht, welche die in ihr schwebenden Kohlenstoffteilchen zur Weissglut bringen. Die hierfür nötige Luftzufuhr wird dadurch erreicht, dass man das Gas unter einem gewissen Druck $(20-30 \mathrm{~mm})$ austreten lässt und im übrigen der Flamme eine hinreichend grosse Oberfläche gibt, so dass reichlich Luft von allen Seiten zu ihr herantreten kann. Diesen Forderungen genügen die sog. Schnittbrenner. Die erste Verbesserung an dem Gaslicht traf Argand mit seinem mit einem Cylinder und zahlreichen Gaseinströmungsöffnungen versehenen Argandbrenner. Seine erhöhte Temperatur drückt sich sofort wieder in seiner zum Gasschnittbrenner, Spektrum 1, etwas vermehrten ultravioletten Strahlung aus (Spektrum 2). Beide, Gasschnittbrenner und Argandbrenner, gehören noch zu den an ultravioletten Strahlen relativ armen Lichtquellen.

Den bedeutendsten Fortschritt auf dem Gebiete der Gasbeleuch- 
tung stellt aber unstreitig die Erfindung des Glühstrumpfes (1885) durch Auer v. Welsbach dar. Alle Spektren der Gaslampen 3-7 zeigen wieder, wie wir dies schon bei dem Petroleum- und Gaslicht nachgewiesen haben, infolge der hohen Temperatur der Glühstrümpfe einen ausserordentlichen Reichtum an ultravioletten Strahlen. Wie die Spektren 3 und 7 an ihrer grösseren Länge erkennen lassen, muss die Temperatur des Glühstrumpfes bei dem stehenden Gasglühlicht eine höhere sein, wie bei dem hängenden Gasglühlicht, Spektrum 4 bis 6. Es ist dies den günstigeren Erwärmungsverhältnissen des Strumpfes und den besseren Verbrennungsverhältnissen des Gases beim stehenden Gasglïhlicht zuzuschreiben.

Die Reihe der Gaslampen wird durch das sich immer mehr ausbreitende Acetylengaslicht geschlossen. Die Spektren 8 und 9 veranschaulichen seinen ausserordentlichen Reichtum an ultravioletten Strahlen, es übertrifft darin alle Gaslampen. Merkwürdigerweise stehen hier bei dem Acetylengaslicht Schnittbrenner und Glühlichtbrenner im umgekehrten Verhältnis zueinander wie Petroleumbrenner und Petroleumglïhlicht oder Gasschnittbrenner und Gasglïhlicht. Während bei diesen das Spektrum des Glühlichtes eine viel grössere Länge als der einfache Brenner hat, zeigt bei dem Acetylengaslicht der Gasschnittbrenner den grösseren Reichtum an ultravioletten Strahlen; sein Spektrum erstreckt sich noch über eine Wellenlänge von $300 \mu \mu$ hinaus. Bei diesen Verhältnissen mag der Einfluss der Glasabsorption bei dem Acetylengasglühlicht (Spektrum 9) eine Rolle spielen.

3. Elektrische Glühlampen. Wenn auch Edison nicht als Erfinder der elektrischen Kohlenfadenglühlampen gelten kann, so gebührt ihm doch unstreitig das grosse Verdienst, die Erfindungen anderer so gestaltet zu haben, dass sie praktisch verwertbar wurden. Im Jahre 1880 kam seine elektrische Kohlenfadenglühlampe zum ersten Male auf den Markt. Sie allein konnte die Konkurrenz mit der gewöhnlichen Gastlamme aufnehmen und die Gasbeleuchtung bis zu einem gewissen Grade verdrängen. Von dem Erscheinen der elektrischen Kohlenfadenglühlampe an begann der scharfe Konkurrenzstreit zwischen Gasbeleuchtung und elektrischer Beleuchtung, der den Erfindungsgeist der Ingenieure auf diesen beiden Hauptgebieten der Beleuchtungstechnik immer zu neuen Anstrengungen um die Vorherrschaft vor der andern anspornte. Diesem Wettkampf ist es vor allen Dingen zu verdanken, dass wir sowohl in der Gasbeleuchtung, sowie in der Beleuchtung durch den elektrischen Strom Lichtquellen 
besitzen, die für einen billigen Preis je nach dem Zweck ein sehr helles Licht liefern.

Während bei den frei brennenden Lampen die in der Flamme schwebenden Kohleteilchen erhitzt werden, werden bei den elektrischen Glühlichtern Kohlenfäden oder Metallfäden vom elektrischen Strom durchflossen und infolge des Widerstandes erhitzt, den sie dem durchfliessenden Strom entgegensetzen. Die dünnen Fäden werden dadurch zur Weissglut gebracht und senden ein blendendes Licht aus.

Taf. VII enthält die Spektren der elektrischen Glühlampen von der Kohlenfadenglühlampe an (Spektrum 1) bis zu der neuesten Metallfadenglühlampe, der Wolframlampe (Spektrum 9).

Alle Spektren zeigen, wie es infolge der hohen Temperatur der Glühfäden auch gar nicht anders zu erwarten war, eine ausserordentliche Ausdehnung im ultravioletten Teil des Spektrums. Zwischen der Kohlenfadenglühlampe (Spektrum 1) und den Metallfadenglühlampen (Spektrum 2-3 und 6--9) lässt sich kaum ein Unterschied nachweisen, sie erstrecken sich alle gleich weit nach der ultravioletten Seite. Eine Ausnahme davon bildet nur die Nernstlampe obne Glocke (Spektrum 4) mit ihrem ausserordentlich langen Spektrum. Die andern Lampen sind alle mit Glocken umgeben. Es lassen gerade diese Spektren erkennen, wie weit gewöhnliches Glas ultraviolette Strahlen absorbiert. Die Grenze liegt etwa bei einer Wellenlänge von $300 \mu \mu$. Noch deutlicher würde sich diese Absorptionsgrenze für gewöhnliches Glas bei etwas längerer Belichtungsdauer hervorheben, oder wenn bei der Aufnahme anstatt des ausserordentlich engen Spaltes von $0,015 \mathrm{~mm}$ ein etwas erweiterter Spalt verwendet werden würde. Insbesondere würde dabei noch schärfer zum Ausdruck kommen, wie schon Spektrum 3, das einer mattierten Tantallampe, erkennen lässt, dass die Mattierung der elektrischen Glübbirnen das Auge vor den ultravioletten Strahlen nicht schützt. Die elektrischen Glühlampen gehören ohne Ausnahme zu den an ultravioletten Strahlen sehr reichen Lichtquellen, ihre ultravioletten Spektren haben noch eine grössere Ausdehnung als die der Gasglïhlichtlampen.

4. Elektrische Bogenlampen. Erst nach Erfindung der Differentialbogenlampe im Jahre 1879 bürgerte sich das elektrische Bogenlicht mehr und mehr ein. Schon frühzeitig wurde man auf den grossen Reichtum des elektrischen Bogenlichtes an ultravioletten Strahlen aufmerksam, glaubte sich aber durch Umhüllung des Licht- 
bogens mit einer Glasglocke genügend gegen die schädliche Einwirkung der ultravioletten Strahlen geschützt. Den trotzdem öfters auftretenden Entzündungserscheinungen durch die Ophthalmia electrica an Personen, die beruflich viel mit elektrischem Bogenlicht zu arbeiten hatten, suchte man durch Abblendung der direkten Lichtstrahlen zu begegnen. Spektrum 1 auf Taf. VIII zeigt die enorm grosse Ausdehnung des Spektrums einer offenen Bogenlampe ohne Glocke. Aber auch mit einer Glocke versehen bleibt die Bogenlampe sehr reich an ultravioletten Strahlen, wie Spektrum 2 erläutert. Das Spektrum wird zwar verkürzt, aber die Absorptionsgrenze für gewöhnliches Glas, wie wir sie im Mittel festgelegt haben, noch überschritten. Und gerade diejenigen Strahlen des ultravioletten Spektrums werden ungehindert durch das Glockenglas hindurchgelassen, an denen das Bogenlampenspektrum infolge seines charakteristischen Bandenspektrums (siehe Spektrum 2) eine grosse Fülle besitzt.

Betrachten wir die andern Spektren von Bogenlampen auf Taf. VIII, wie sie heute überall zur Beleuchtung verwandt werden, so können wir an der Bogenlampe mit eingeschlossenem Lichtbogen, oft auch Dauerbrandbogenlampe genannt, Spektrum 3 und 4, sowie an den Flammenbogenlampen, einerlei ob sie mit weissen oder gelben Effektkohlen brennen (Spektren 5, 6 und 7), die gleiche Beobachtung machen.

Als letzte unserer künstlichen Lichtquellen ist noch eine Lampe, die sich immer mehr einbürgert und erhöhte Bedeutung gewinnt, zu erwähnen: die Quecksilberdampflampe. Gerade sie wird heute mit grossem Pathos als die beste Lichtquelle für Räume, wie Zeichenund Schulsäle, überhaupt für alle Räume, in denen das Auge durch feine Arbeiten besonders angestrengt wird, angepriesen. Begründet wird dies mit ihrem eigentümlichen, fast einfarbigen Licht, das die Kontraste schärfer hervortreten lässt. Spektrum 8 auf Taf. VIII zeigt das charakteristische Linienspektrum einer Quarzquecksilberdampflampe ${ }^{1}$ ). Es lässt erkennen, dass sie mit ibrem Anteil an schädlichen ultravioletten Lichtstrahlen nicht hinter den elektrischen Bogen* lampen zurückbleibt, sondern sie noch eher übertrifft. Da die Lampe selbst sehr arm an roten und gelben Lichtstrahlen ist, so tritt ihr

1) Es ist aus dem Buche: K. Stockhausen, Der eingeschlossene Lichtbogen bei Gleichstrom, Verlag von J. A. Barth, Leipzig 1907 entnommen und war ihm von Herrn Dr. H. Thiele zur Verfügung gestellt. 
grosser Anteil an der photochemischen Strahlung nur um so schärfer hervor. - Die Spektren 3-7 sind mit einem $4 \mathrm{~mm}$ breiten Querspalt und auf anderen Platten aufgenommen.

Fassen wir unsere Untersuchungen über den Reichtum unserer künstlichen Lichtquellen an ultravioletten Strahlen nochmals kurz zusammen, so können wir drei Klassen unterscheiden, und zwar Lichtquellen, die
1. arm,
2. relativ arm,
3. reich

an ultravioletten Strahlen sind.

Zur ersten Klasse gehören: die römische Olivenöllampe, die Kerzen und die offenen Rüböllampen; zur zweiten: die Rüböllampen mit Cylinder, die Petroleumschnitt- und Rundbrenner, die Gasschnittbrenner und die Gasargandbrenner. In die dritte Klasse gehören alle Lampen, die mit einem Glühstrumpf ausgerüstet sind, wie Petroleumund Spiritusglühlampen, hängende und stehende Gasglühlichtlampen. Noch reicher an ultravioletten Strahlen als diese sind aber das Acetylenlicht, alle elektrischen Glühlampen, wie Kohlenfadenglühlampen, Nernstlampen, Metallfadenglühlampen und besonders alle Bogenlampen und Quecksilberdampflampen.

II. Der schädliche Einfluss der ultravioletten Strahlen auf das Auge.

Über den Einfluss der ultravioletten Strahlen auf das Auge besitzen wir eine Reihe sehr sorgfältiger Dntersuchungen; vor allen waren es die Arbeiten von Widmark, Schulek, Hertel, BirchHirschfeld, Herzog, Hess, die unsere Kenntnisse därin wesentlich gefördert haben. Wir suchten uns aber auch selbst in dieser Frage ein Urteil zu bilden. Im elektrotechnischen Institut der Dresdener technischen Hochschule wurde uns dazu Gelegenheit geboten, und es drängt uns, Herrn Prof. Görges dafür unsern verbindlichsten Dank auch hier auszusprechen. Wir wiederholten zunächst die Versuche von Widmark. Derselbe hatte zwischen der elektrischen Bogenlampe und dem Auge des Versuchstieres bei der einen Reihe der Versuche eine Bergkrystalllinse eingeschaltet, bei der andern schob er ausserdem noch eine $2{ }^{1}{ }_{2} \mathrm{~mm}$ dicke Fensterglasscheibe ein. Im ersten Fall bekam er die Erscheinungen einer äusserst heftigen elektrischen Ophthalmie, im zweiten Fall wurde das Auge nur wenig beeinflusst. Wir bekamen bei unseren Versuchen auch deutliche Unterschiede, je nachdem wir das Licht durch Quarz allein oder durch Quarz und eine Glasscheibe leiteten, uur konnten wir nicht finden, dass Glas alle Reizerscheinungen aufhob, die man den ultravioletten Strahlen 
zuschreibt. Wir haben Tafelglasplatten von $18 \mathrm{~mm}$ Durchmesser eingeschaltet und bei der an ultravioletten Strahlen ganz besonders reichen Kopierbogenlampe mit eingeschlossenem Lichtbogen (für 10 Amp.), mit der wir unsere Versuche zum grössten Teil ausführten, ganz erhebliche Erscheinungen am belichteten Auge gefunden. Widmark sah übrigens auch solche Erscheinungen, und nur weil sie verhältnismässig gering waren, legte er ihnen weniger Bedeutung bei. Wir haben die Versuche mit der erwähnten Kopierlampe vier Stunden lang fortgesetzt und bekamen, auch wenn die dicke Glasplatte eingeschaltet war, katarrhale Symptome der Conjunctiva der Lider, Injektion und starke Chemosis der Conjunctiva bulbi, Epithelabhebung und Trübung der Hornhaut, Pupillenverengung und Verfärbung der Iris. Wurde die Glasplatte und die Glasglocke weggelassen, so kamen dieselben Erscheinungen rascher und intensiver zur Entwicklung. Dieser Tierversuch zeigte also, dass die Reizerscheinungen, die den ultravioletten Strahlen zugeschrieben werden, auch von einer starken Glasplatte nur abgeschwächt, aber nicht ganz aufgehoben werden. Es lehrte also der Tierversuch dasselbe wie der eingangs angeführte Krankheitsfall, bei dem die Lampenglocke und die Brille des Patienten nicht gèügend vor den schädlichen Wirkungen dieser Strahlen schützten.

Die Erscheinungen der elektrischen Ophthalmie sind bei den Elektrotechnikern heute bekannt, sie werden, da sie die verhältnismässig gute Prognose kennen, dadurch nicht mèhr besonders beunruhigt. Fälle, bei denen auch Veränderungen in der Netzhaut auftreten und Störungen dauernd zurückbleiben, sind selten, und da ist es noch zweifelhaft, ob diese Störungen nicht allein auf die leuchtenden Strahlen bei zu grosser Lichtstärke zu beziehen sind. Wir sehen solche Störungen eigentlich nur noch bei Sonnenlicht, wenn bei Gelegenheit einer Sonnenfinsternis direkt in die noch teilweise verdeckte Sonne hineingeblickt worden ist. Die anderen Erscheinungen der elektrischen Ophthalmie sind aber, wie schon eingangs erwähnt, auch bei anderen Lichtquellen, die reich an ultravioletten Strahlen sind, je nach dem Gehalt an diesen mehr oder weniger stark ausgeprägt. Dasselbe klinische Bild wie bei der Ophthalmia electrica finden wir bei Schneeblindheit, wo die von der Schneefläche reflektierten ultravioletten Strahlen des Sonnenlichtes die Schuld tragen. Auch die Erscheinungen der Erythropsie sind auf die Wirkung der ultravioletten Strahlen zu beziehen. Es ist also das Sonnenlicht, vor allem das direkte oder direkt reflektierte unter Umständen gar nicht 
so harmlos, wie man meistens meint. Aber auch unsere künstlichen Lichtquellen, die wir täglich zur Beleuchtung unserer Wohnräume und Arbeitsplätze verwenden, sind nicht frei von solchen Wirkungen. Uns persönlich ist als Arbeitslampe immer eine gute Petroleumlampe mit Rundbrenner lieber gewesen als das beste Auerlicht oder elektrisches Licht. Es liegt dies sicher daran, dass dieses Petroleumlicht nicht so reich an ultravioletten Strablen ist, wie jene. Jeder von uns merkt, wenn er ein Arbeitsquantum, das er bei Tageslicht gerade noch ohne eine Anstrengung seiner Augen zu empfinden, ausführen kann, plötzlich bei licht ausführen muss, dass seine Augen rascher ermüden. Das Licht drückt ihm auf die Augen. Noch auffälliger ist dies, wenn an den Augen schon katarrbale Symptome bestehen. Wieviel chronische Bindehautkatarrhe machen den Patienten erst Beschwerden, wenn im Herbst die Arbeit bei Lampenlicht beginnt? Das diffuse Tageslicht hat sie nicht belästigt, eben weil bei diesem durch die Absorption der Atmosphäre und durch mehrfache diffuse Reflexion, bevor es auf unsern Arbeitsplatz gelangt, ein grosser Teil der ultravioletten Strahlen verloren gegangen ist.

Die Reizerscheinungen am äusseren Auge, die wir bei der Arbeit bei künstlichem Licht bekommen, sind sicher die Wirkung der ultravioletten Strahlen; sie sind nichts anderes als die allerersten Anfänge des als elektrische Ophthalmie bezeichneten Krankenbildes.

Dass die uitravioletten Strahlen bis zur Netzhaut gelangen, und auch diese zu reizen vermögen, ist bekannt. Wir wissen, dass bei Staroperierten das Spektrum nach der ultravioletten Seite hin verlängert ist, wir wissen, dass das Rotsehen (Erythropsie), an dem Staroperierte leicht leiden, veranlasst wird durch die Finwirkung der ultravioletten Strahlen auf die Netzhaut. Fuchs und Andere haben ferner das Rotsehen, das sich bei normalen Augen zeigt, wenn man nach einer längeren Gletscherwanderung in eine dunkle Hiutte tritt, als eine Wirkung der ultravioletten Strahlen auf die Netzhaut erkannt. Birch-Hirschfeld hat experimentell diese Frage geprüft. Danach erzeugen diese Strahlen auch Veränderungen in der Netzhaut, die zwar nicht mit dem Augenspiegel sichtbar sind, die aber nach mikroskopischen Untersuchungen in Auflösung der Chromatinsubstanz der Ganglienzellen und Auftreten von Vakuolen im Protoplasma derselben, Chromatinverlust der. Körner der inneren, Ausbleichung und Formveränderung der Körner der äusseren Körnerschicht bestehen. Solche Veränderungen konnte er aber nur nachweisen, wenn die Linse aus dem Auge entfernt war. Er hat damit 
den Nachweis erbracht, dass der Linse die wichtige Rolle zukommt, die Netzhaut vor der Einwirkung der ultravioletten Strahlen zu schützen. Dass die Linse diese Rolle spielt, war schon von Widmark erkannt worden. Derselbe sah, dass die Linse bei der Beleuchtung. mit solchen Strahlen intensiv fluoresziert. Wir kannten diese Arbeiten Widmarks und waren doch überrascht, als wir zum ersten Male sahen, wie intensiv bei unserer Kopierlampe dieses Phänomen hervortrat.

Wenn Strahlen gewissẹr Wellenlänge (hier die ultravioletten) einen Körper treffen, der die Eigenschaft hat zu fluoreszieren, so wird die Energie, die solchergestalt dem Körper zugeführt wird, wieder abgesandt, in leuchtende Strahlen umgesetzt. Bei dieser Erwägung drängt sich unmittelbar der Gedanke auf: Werden dabei in dem Organ durch diese Umsetzung der Energie mit der Zeit gar keine Veränderungen hervorgerufen? Widmark, Herzog, BirchHirschfeld beobachteten Iinsentrübungen, wenn sie die Linse aus dem Versuchsauge herausnahmen und mit dem Kontrollauge verglichen. Hess hat anatomische Veränderungen an der Linsenkapsel nachgewiesen. Könnte der Altersstar nicht als ein Produkt dieses Prozesses gedeutet werden? Diese Frage ist jetzt diskutabel geworden. Man hat schon immer versucht, die Starbildung als eine Folge der Lichteinwirkung auf das Auge hinzustellen. Könnte nicht der ultraviolette Anteil dabei der wichtigste Faktor sein? Man wird freilich sagen: Der Altersstar beginnt in der Peripherie der Linse, in Partien, die durch die Regenbogenhaut vor der direkten Einwirkung der ultravioletten Strahlen geschützt sind. Dieser Umstand liesse sich aber vielleicht daraus erklären, dass in der Linse gleichzeitig noch ein anderer Prozess vor sich geht, der es unmöglich macht, dass auch im Zentrum der Linse diese Veränderungen auftreten. Es ist dies die Sklerose des Linsenkerns. Der Linsenkern fängt schon in den vierziger Jahren an hart und derb zu werden, an ihm kann keine solche Veränderung auftreten. Die Trübung ist daher nur an den noch nicht durch den sklerosierenden Prozess veränderten Teilen möglich. Wird die Linse in einem Alter, wo dieser sklerosierende Prozess noch nicht eingesetzt bat, der Einwirkung ultravioletter Strablen besonders ausgesetzt, so triibt sich gerade das Zentrum der Linse, wie wir dies beim Glasmacherstar sehen, wo sich ein dunkler Strang in der Linse bildet, der von hinten nach vorn durch die Linse zieht und mit der Zeit das ganze Pupillargebiet ausfüllt, während die peripheren Partien noch vollständig klar 
sind. Wenn man den Altersstar als eine Abnutzung des Schutzorganes gegen die Wirkung der ultravioletten Strahlen hinstellen will, müisste man den Nachweis erbringen, dass jetzt, wo man bei viel intensiveren Lichtquellen arbeitet als früher, auch diese $\mathrm{Ab}$ nutzungserscheinungen eher auftreten. Der Beweis wird so leicht nicht zu erbringen sein, zumal es sich um einen Prozess handelt, der sich erst im Laufe des Lebens abspielt und erst gegen das Ende desselben zu solchen Erscheinungen führen könnte. Beachtlich ist aber dabei, dass Schulek bewiesen hat, dass die Fluorescenz der Linse im Laufe des Lebens abnimmt. Und bei unsern Tierversuchen haben wir gesehen, dass die Fluorescenz mit der Dauer der Belichtung (deutlich ausgesprochen nach etwa 4 Stunden) geringer wird und dass auch am nächsten Tag die Fluorescenzerscheinung noch nicht wieder so intensiv hervortritt wie bei einem Tier, dass in der letzten Zeit solch intensiver Beleuchtung nicht ausgesetzt worden ist. Es ist damit zweifellos erwiesen, dass nicht nur im Laufe des Lebens, sondern auch bei intensiver Belichtung mit ultraviolettem Licht in wenigen Stunden eine Veränderung des Stoffes entsteht, der die Fluorescenz der Linse bewirkt. Ausserdem haben wir gefunden, dass beim Altersstar der sklerosierte Kern sehr viel weniger intensiv fluoresziert als die noch nicht sklerosierten Linsenpartien.

Dass auch im normalen menschlichen Auge die Schutzkraft der Linse in wenig Stunden geringer werden kann, ergibt sich aus den Untersuchungen über die Erythropsie.

Wir wissen vor allen durch die Untersuchungen von Fuchs iiber das Rotsehen (Erythropsie), das auftritt, wenn man nach längeror Gletscherwanderung in eine dunkle Hütte tritt, dass dies bedingt ist durch die Einwirkung der ultravioletten Strahlen auf die Netzhaut. In der Ebene finden sich diese Erscheinungen nur bei Personen, denen die Linse entfernt ist und die eine durch die Operation erweiterte Pupille haben. Im Hochgebirge tritt dieselbe Erscheinung auch an normalen Augen ein, wenn man längere - Zeit über Schneeflächen gewandert ist. Die Erklärung dieser Erscheinung ergibt sich aus der Schutzkraft der Linse gegenüber der Wirkung der ultravioletten Strahlen. In der Ebene ist das Tageslicht ärmer an ultravioletten Strahlen als in Gletscherhöhe. Die Atmosphäre absorbiert einen grossen Teil davon. In der Ebene kann das ultraviolette Licht nur in den Augen eine Reizung der Netzhaut erzeugen, in denen das Schutzorgan (die Linse) entfernt ist. Anders ist 
es im Hochgebirge, dort ist das Licht reicher an ulltravioletten Strahlen. Zudem reflektiert Schnee, vor allem gefrorener Schnee, die ultravioletten Strahlen ganz vorziglich. Bei kurzdauernder Einwirkung auf das Auge erzeugt das Licht keine Reizung der Netzhant, weil die Stoffe, welche die Fluorescenz der Linse erzeugen, die ultravioletten Strahlen, nicht zur Netzhaut gelangen' lassen. Erst nach länger anhaltender Einwirkung sind diese Stoffe aufgebraucht, die Linse gleichsam daran ausgebleicht. Damit verliert sie ihre Schutzkraft. Die ultravioletten Strahlen gelangen zur Netzhaut und erzeugen das Rotsehen (Erythropsie). Fs spielt sich hier im normalen Auge genau derselbe Prozess ab, den wir bei unseren Versuchstieren nach mehrstündiger Belichtung beobachten konnten. Das Ausbleichen der Linse muss daher als ein physiologischer Vorgang gelten ${ }^{1}$ ).

Durch Tierexperimente wissen wir jetzt, dass die ultravioletten Strahlen in der Linse Stoffumsetzungen veranlassen; wir wissen, dass bei längeren Belichtungen mit ultravioletten Strablen leichte Trübungen der Linse auftreten, und wir wissen schliesslich, dass sich in solchen Linsen mikroskopische Veränderungen in der Linsenkapsel nachweisen lassen, und durch Erfahrungen in der Praxis wissen wir, dass bei Menschen, die der Einwirkung eines an ultravioletten Strahlen besonders reichen Lichtes viel ausgesetzt sind, wie die Glasmacher, eigentümliche Trübungen der Linse auftreten. Muss sich da nicht die Frage aufdrängen, stehen die Trübungen der Linse, die mit zunehmendem Alter eintreten, nicht auch damit im Zusammenhang? Die Fluorescenz der Linse und der damit zusammenhängende Stoffumsatz in der Linse ist ein physiologischer Vorgang, der sich tagtäglich abspielt, könnte er nicht am Ende des Lebens zum Zerfall der Linsenfasern und zur Trübung der Linse (Starbildung) die Veranlassung geben? Durch die intensiven künstlichen Lichtquellen ist unsere Generation mehr als frïhere der Einwirkung der ultravioletten Strahlen ausgesetzt. Wir haben oben absichtlich gezeigt, dass wir die Entwicklung dieser Lichtquellon mit durchgemacht haben und dass zum erstenmal an uns diese Erscheinungen zum Ausdruck kommen könnten. Der Nachweis, dass der Star jetzt häufiger geworden ist, wird so leicht nicht zu erbringen sein, da vergleichend statistisches Material über die Häufigkeit des Stares nicht zu beschaffen ist. Unter diesen Umständen dürften aber schon einzelne gut beobachtete Fälle, bei denen eine intensive Einwirkung mit ultravioletten Strahlen zur Linsentrübung

1) Die abweichende Ansicht A. Vogts (Arch. f. Augenheilk. Bd. LX. S. 91) wird auf S. 69 unserer Arbeit noch kurz berithrt werden. 
Veranlassung geben konnte, beweiskräftig sein. Ein solcher Fall sei hier mitgeteilt: Ein Herr, Mitte der vierziger Jahre, der aus einer Familie stammt, in der frühzeitige Erkrankungen an Star nicht vorgekommen sind, hat in seinem Leben 25-30 schwere elektrische Ophthalmien durchgemacht. Er ist in seiner Jugend wiederholt wegen seines starken Astigmatismus und auch bei seinen ersten elektrischen Ophthalmien von verschiedenen ophthalmologischen Autoritäten untersucht worden. Es wurden bei keiner Untersuchung Trübungen der Linse festgestellt. Der Patient ist ein äusserst exakter Beobachter und mit optischen Dingen wohl vertraut. Er hätte diese Trübungen sicher in früheren Jahren gemerkt. Jetzt hat er Trübungen in der Linse und zwar sitzt die Haupttribung in dem Pupillargebiet hinter der vorderen Linsenkapsel und schneidet nach oben in einer horizontalen Linie $a b$, die etwa dem oberen Lidrand bei halbzugekniffenen Lidern entsprechen würde. In der Peripherie der Linse befinden sich ausserdem streifenförmige, bis in das Pupillargebiet reichende Trübungen. Dieselben sind beiderseits in der unteren Hälfte der Linse stärker ausgeprägt als in der oberen. Könnte eine solche frühzeitige Startrübung nicht eine Folge der 25-30 elektrischen Ophthalmien sein, die der Patient durchgemacht hat? Der Fall mahnt dringend zur Beachtung, und wir müssen uns daher nicht nur wegen den Reizerscheinungen, welche die nltravioletten Strahlen auf die äusseren Augenteile ausüben, sondern auch wegen der Möglichkeit, dass die Bildung des Altersstars durch sie beschleunigt wird, die Frage vorlegen: „Wie können wir unsere Augen vor den Einwirkungen dieser Strahlen schützen?" Für den Sehakt werden dieselben nicht gebraucht und es liegt nahe, diese Strahlen durch Filter, welche die sichtbaren Strahlen nicht wesentlich schwächen, abzufangen. Wenn man gemeint hat, dass Glas; das als Lichthülle verwandt wird, das schon in zureichendem Masse tue, so beweist der eingangs erwähnte Fall und auch der astigmatische Elektrotechniker mit seinen 25-30 Ophthalmien, dass eine Glasbrille und die üblichen Lampenglocken keinen genügenden Schritz bieten. Bis jetzt hat die Industrie nichts getan, um diese Strahlen von dem Auge fern zu halten. Und es war vorerst nötig, zu prüfen, ob es Glassorten gibt, die solche Strahlen in stärkerem Masse oder vollständig abhalten und die sichtbaren Strahlen in ausreichender Weise hindurchlassen. Dieses wurde der Gegenstand einer weiteren Untersuchung. 
Wie schützen wir unsere Augen v. d. Einwirk. d. ultraviol. Strahlen usw.

\section{Die Absorptionsfähigkeit der Glassorten gegenüber ultravioletten Strahlen.}

Da feststeht, dass unsere künstlichen Lichtquellen an ultravioletten Strahlen immer reicher geworden und diese Strahlen für unser Auge schädlich sind, so war nun die Frage zu erörtern, ob wir nicht Mittel besitzen, das Licht unserer künstlichen Lichtquellen von den schädlichen ultravioletten Strahlen zu befreien. Es war zu prïfen, ob und wie weit die vorhandenen Glassorten, die als Cylinder, Glühlampenbirnen, Lampenglocken oder als Schutzbrillen in Gebrauch sind, die ultravioletten Strahlen absorbieren. Weitere Beobachtungen mussten sich auf den Einfluss, den die Dicke des Glases auf den ultravioletten Teil des Spektrums ausübt, erstrecken.

Bei allen Untersuchungen über die Absorptionsfähigkeit der Glassorten gegenüber ultravioletten Strahlen diente eine Wechselstrombogenlampe von $10 \mathrm{Amp}$. als spektrale Lichtquelle.

Über die Durchlässigkeit der heute im Gebrauch befindlichen Beleuchtungsgläser gegenüber ultravioletten Strahlen geben die Taf. IX und $\mathrm{X}$ Auskunft. Sie lassen erkennen, dass alle Beleuchtungsgläser aus Klarglas den ultravioletten Strahlen in reichem Masse den Durchgang gestatten. Auch der Glimmercylinder, der vielfach bei dem Gasgliblicht Verwendung findet, bietet den ultravioletten Strahlen des Gasglühlichtes kein Hindernis.

A. Staerkle ${ }^{1}$ ) schlägt vor, die Glasumbüllungen bei den Lichtquellen mit dickeren Wandstärken herzustellen, um die Schädlichkeit unserer modernen Lichtquellen zu vermindern. Er schreibt: „Dieselbe kann auf eine Wandstärke von $3-4 \mathrm{~mm}$ gebracht werden, ohne eine merkbare Abschwächung der Lichtintensität herbeizuführen gegenüber den jetzt gebräuchlichen Gläsern mit einer Wanddicke von $1 \mathrm{~mm}$ oder nur wenig darüber."

Abgesehen von technischen und physikalischen Schwierigkeiten, die sich dieser Forderung entgegenstellen, lassen unsere Untersuchungen auf Taf. IX klar erkennen, dass mit solchen Massnahmen nur wenig erreicht würde. Beleuchtungsgläser von einer Wandstärke von $0,4 \mathrm{~mm}$ und $1 \mathrm{~mm}$ (Spektrum 2, Klarglas von einer Glühlampenbirne, Spektrum 3, Klarglas von einer Bogenlampenglocke, und Spektrum 7, Gasglühlichtcylinder) ïberschreiten die Glasabsorptionsgrenze für gewöhnliches Glas, die wir im Mittel bei der Wellenlänge von $300 \mu \mu$

1) A. Staerkle, Über die Schädlichkeit moder.zer Lichtquellen auf das Auge und deren Verhütung. Arch. f. Augenheilk. Bd. L. S. 121.

7. Graefe's Archiv für Ophthalmologie, LXIX. 1. 
festgelegt haben, um ein beträchtliches. Die Absorptionsgrenze liegt bei diesen bei der Wellenlänge $290 \mu \mu$. Wird die Glasdicke auf $1,5 \mathrm{~mm}$ erhöht, so tritt eine geringe Absorption ein und zwar bis zur Wellenlänge $300 \mu \mu$. Aber erst bei einer Glasplatte von $9 \mathrm{~mm}$ tritt eine stärkere Absorption ein, so dass nur noch Strahlen mit einer grösseren Wellenlänge als $320 \mu \mu$ der Durchgang gestattet ist. Wird die Glasdicke auf $18 \mathrm{~mm}$ erhöht, so können Strahlen von kleinerer Wellenlänge als $345 \mu \mu$ nicht mehr hindurchtreten.

Staerkle ist weiter der Meinung, Gläser mit hohem Bleigehalt müssten sich durch eine starke Absorption der ultravioletten Strahlen auszeichnen. Das ist aber nicht der Fall, wie Spektrum 2 auf Taf. X erkennen lässt. Es ist dies ein Absorptionsspektrum einer Brille aus Bleiglas mit sehr hohem Bleigehalt, wie solche bei Röntgenaufnahmen zum Schutze der Augen gegen Röntgenstrahlen getragen werden. Das ultraviolette Spektrum erstreckt sich zwar nicht bis ganz an die Glasabsorptionsgrenze von gewöhnlichem Glas, es ist dies aber mehr der verhältnismässig grossen Glasdicke von $4 \mathrm{~mm}$ als dem Bleigehalt zuzuschreiben. Ein gewöhnliches Brillenglas von $2 \mathrm{~mm}$ Dicke lässt alle ultravioletten Strahlen bis zu einer Wellenlänge von $290 \mu \mu$ ungehindert hindurchtreten. Es unterscheidet sich in nichts von dem der Petroleum- und Gasglühlichtcylinder.

Dass auch die verschiedenen Opalin-, Opal-, Matt- und Milchgläser nicht unsere Lichtquellen von den schädlichen ultravioletten Strablen befreien, wie oft fälschlich gemeint wird, lassen die Spektren 3-7 auf Taf. $X$ zur Genüge erkennen.

Nachdem sich also gezeigt hatte, dass unter den Beleuchtungsgläsern kein Glas vorhanden ist, das die ultravioletten Strahlen in genügender Weise abhält, haben wir versucht, ob die Gläser, die als Schutzbrillen gebräuchlich sind, vielleicht solche Eigenschaft besitzen.

Zunächst haben wir die blanen Brillengläser geprïft. Die verschieden stark blau gefärbten Brillen, Spektren 1-4 auf Taf. XI, sind gerade das Gegenteil von Schutzgläsern; sie lassen auch in ihren stärksten Nummern gerade die ultravioletten Strahlen besonders gut hindurch. Selbst zwischen der stärksten und der schwächsten Nummer lässt sich in bezug auf die Ausdehnung des ultravioletten Spektrums kaum ein Unterschied wahrnehmen.

Die rauchgrauen Gläser, Spektren 5-8, sind schon etwas besser. Sie schwächen das ganze Spektrum gleichmässig. In den dunkelsten fast schwarzen Nummern ist das ultraviolette Spektrum deutlich verkïrzt. 
Auf Taf. XII befinden sich Spektren anderer Gläser, die als Schutzbrillen Verwendung gefunden haben. 1-3 Gläser blen ardoise zeigen dasselbe an ultravioletten Strahlen reiche Absorptionsspektrum wie die gewöhnlichen blauen Schutzbrillen. Auch Rosalinglas, Spektren 4-6, hat auf die ultravioletten Strahlen keinen schützenden Einfluss. Nur gelbe Gläser, wie sie als Jagdgläser im Handel zu haben sind, verkürzen etwas das ultraviolette Spektrum (Spektrum 7 und 8).

Andere im Gebrauche befindlichen Brillengläser sind die Fieuzalund Enixanthosgläser. Van Genderen Stort') wies experimentell nach, dass die Pigmentwanderung und die Bewegung der retinalen Elemente unter gelbem Licht im Minimum stattfinden. Auf Grund dieser Untersuchungen stellte Fieuzal ${ }^{2}$ ) vor 20 Jahren Schutzbrillen aus grau-grünlich-gelbem Glas her. Eine Abhaltung der ultravioletten Strablen von dem Auge durch Gebrauch dieser Gläser beabsichtigte er nicht. Damals war die Schädlichkeit der kurzwelligen Strahlen für das Auge auch noch gar nicht bekannt.

Schulek hat diese Gläser schon auf ihre Durchlässigkeit für ultraviolette Strahlen geprüft und fand, dass sie dieselben schwächen, aber nicht ganz auslöschen. Unsere Untersuchungen führten zu demselben Ergebnis (siehe Taf. XIII, Spektren 1-3). In den schwächsten Nummern lassen sie noch einen erheblichen Teil ultravioletter Strahlen hindurch. In den stärksten Nummern sind sie so dicht, dass sie zwar die ultravioletten Strahlen absorbieren, aber auch gleichzeitig die leuchtenden Strahlen so schwächen, dass sie für Beleuchtungsgläser nicht zu gebrauchen sind. Staerkle ${ }^{3}$ ) und Hallauer ${ }^{4}$ ) haben diese Eigenschaften des Fieuzalglases erkannt und haben dieses für Schutzbrillen besonders empfohlen. Da wo gleichzeitig eine starke Schwächung der sichtbaren Strahlen erwünscht ist, sind diese Gläser sicher von den jetzt existierenden die besten. Von dem Enixanthosglas

1) Van Genderen Stort, Über Form- und Ortsveränderungen der Netzhautelemente unter Einfluss von Licht und Dunkel. Graefe's Arch. f. Ophth. Bd. XXXIII, 3. 1887.

2) Fieuzal, Les verres gris-jaunes et les monvements des éléments rétiniens, Bulletin de la clinique nationale ophthalmologique de l'hospice des QuinzeVingts. Tome V. No. 2. 1887. - Des verres colorés en hygiène oculaire. Daselbst. Tome III. No. 3. 1885.

3) A. Staerkle, Über die Schädlichkeit moderner Lichtquellen auf das Ange und deren Verhütung. Arch. f. Angenheilk. Bd. L. S. 121.

4) 0. Hallauer, Einige Gesichtspunkte für die Wahl des Brillenglasmaterials. Ber. der ophth. Ges. Heidelberg 1907. 
gilt dasselbe. In seiner schwächsten Nummer (Spektrum 4) lässt es noch erheblich ultraviolettes Licht durch, in der stärksten Nummer (Spektren 5 und 6) löscht es nur die ultravioletten Strahlen grösserer Wellenlänge aus und schwächt dabei das sichtbare Spektrum.

Unsere Untersuchungen hatten uns ergeben, dass unter den vorhandenen Lampen- und Beleuchtungsgläsern sowie unter den Schutzbrillen kein Glas existierte, das unsern Forderungen genügte. Hatten wir es doch zu unserer Aufgabe gemacht, ein Glas aufzufinden oder neu herzustellen, das die ultravioletten Strahlen vollständig absorbieren, dabei aber die leuchtenden Strahlen möglichst wenig schwächen sollte.

Es bestand nun noch die Möglichkeit, dass ein solches Glas, das unsere Anforderungen erfüllte, schon in irgend einer Form vorhanden war, an dem aber diese Eigenschaften noch nicht erkannt worden waren. Am nächsten lag es, die photographischen Lichtfilter auf ihre Durchlässigkeit gegenüber ultravioletten Strahlen zu prüfen. Spektrum 8 und 9 stammen von zwei solchen Filtern und lassen erkennen, wie wenig diese den ultravioletten Teil des Spektrums absorbieren.

Wir setzten uns darauf mit verschiedenen Glashütten, Farbenglaswerken, Ateliers für Glasmalereien und dgl. in Verbindung und erhielten von diesen ganze Glasskalen der verschiedensten Färbungen. Ausserdem kauften wir in Glashandlungen Gläser aller Färbungen in grosser Anzahl auf. Diese Gläser wurden alle einer genauen Untersuchung unterworfen, die aber fast durchweg negative Resultate ergab. Anfragen an die betreffenden Glaswerke über die Zusammensetzung einiger Gläser blieben in fast allen Fällen erfolglos, da die Fabriken die Zusammensetzung ihrer Gläser als Fabrikgeheimnis hüten. Die Untersuchung aller dieser Gläser, die so lichtdurchlässig waren, dass sie als Beleuchtungsgläser in Frage kommen konnten, ergab, dass sie nur die ultravioletten Strahlen von geringer Wellenlänge abhalten (geringer als $300 \mu \mu$ ). Diese Grenze schwankt je nach der Dicke des Glases. Solche Gläser bieten aber nur einen geringen Schutz gegen die Wirkung der ultravioletten Strahlen auf den Organismus. Aus der Verwendung der ultravioletten Strahlen zur Behandlung von Hautkrankheiten wissen wir, dass die ultravioletten Strahlen kürzester Wellenlänge zwar leicht oberflächliche Entzündungserscheinungen hervorrufen, aber nicht so weit in die Tiefe der Gewebe eindringen wie die ultravioletten Strahlen grösserer Wellen- 
länge. Wenn wir dem Auge einen Schutz bieten wollen, so müssen wir vor allem die Strahlen von $400 \mu \mu$ bis $300 \mu \mu$ abfangen. Da wir ein solches Glas nicht fanden, blieb uns nichts anderes iubrig, als uns in einem eignen Laboratorium selbst mit dem eingehenden Studium der Glastechnik, besonders aber mit der Herstellung und $\mathrm{Zu}$ sammensetzung der Gläser zu befassen.

Nach langen Bemühungen und zahlreichen Schmelzversuchen gelang es uns, nachdem wir beinahe an einem brauchbaren Erfolg unserer Mühen zweifelten, die Herstellung eines Glases, das alle ultravioletten Strahlen vollständig auslöscht. Das Glas hat eine schwachgelbgriune Farbe und die leuchtenden Strahlen werden bei einer Dicke von $0,5-1 \mathrm{~mm}$ nur wenig durch dasselbe geschwächt (etwa $5 \%$ ). Es wird als Euphosglas in den Handel kommen. Es ist zum Patent angemeldet und Name und Warenzeichen geschützt. Bemerkt sei hier noch, dass die Farbe des Glases an sich nicht das Wirksame ist. Der Farbenton lässt sich mit jedem Glassatz erzeugen, damit erlangt aber das Glas noch nicht die Eigenschaft, die ultravioletten Strahlen abzufangen.

Das Spektrum 10 auf Taf. XIII zeigt das Absorptionsspektrum des Euphosglases. Es lässt klar erkennen, dass nur die sichtbaren Strahlen durch das Glas hindurchtreten können, alle unsichtbaren, ultravioletten Strahlen aber werden von ihm versehluckt. Auch bei sehr langer Belichtungszeit verlängert sich das Spektrum nicht nach der ultravioletten Seite, wie Spektrum 3 auf Taf. XIV veranschaulicht.

Nachtrag: Dieser Abschnitt unserer Untersuchung war zur Zeit der Naturforscherversammlung in Dresden bereits abgeschlossen und haben wir in der gemeinsamen Sitzung der hygienischen und ophthalmologischen Sektion darüber berichtet und Proben des Euphosglases vorgezeigt. Durch das Referat, das davon in den Zeitungen erschien, angeregt, hat Vogt (Arch. f. Augenheilk. Bd. LIX) eine vorläufige Mitteilung über ein Glas gebracht, das dieselben Figenschaften haben sollte und das er von Schott und Gen. in Jena bezogen hatte. Wir hatten uns bei Beginn unserer Untersuchungen deshalb auch an diese Firma gewandt und zwei verschiedene Gläser erhalten, die diese Eigenschaft haben sollten. Die genauere Untersuchung ergab, dass sie wohl etwas mehr wie die übrigen Gläser, aber lange nicht für unsere $Z$ wecke ausreichend diese Eigenschaft besassen. Wir haben uns auch das von Vogt empfohlene Glas verschafft und gefunden, dass es nur in Dicken von $6-8 \mathrm{~mm}$ genügend absorbiert. Ein derartig dickes Glas ist schon aus technischen Grün- 
den als Beleuchtungsglas schwer verwendbar. Vogt scheint auch erkannt zu haben, dass es nur in dicken Schichten genügend wirkt. In seiner letzten Publikation hat er ein $9 \mathrm{~mm}$ dickes Stück desselben zu seinen Versuchen über die Entstehung der Erythropsie verwandt.

Bei dieser Gelegenheit sei schon jetzt darauf aufmerksam gemacht, dass Vogt bei dieser Publikation: Zur Frage der Entstehung der Erythropsie (Arch. f. Augenheilk. Bd. XL) die Erythropsie wahrscheinlich mit den Erscheinungen des komplimentär gefärbten Nachbildes verwechselt hat. Er erweiterte sich die Pupille eines Auges mit Euphthalminkokain und erzeugte sich angeblich durch Fixation einer sonnenbeschienenen Schneefläche (nach dem Vorgang von Fuchs) Erythropsie: „das Rotsehen trat schon nach wenigen (10-15) Sekunden auf, sofern der Blick von der Schneefläche weg in einen mässig erleuchteten Raum gewendet wurde, und dauerte mehrere Minuten." Nach den Angaben von Fuchs (Graefe's Arch. Bd. XLII) tritt die Erythropsie unter solchen Bedingungen nur auf, wenn das Auge „recht lange" dem Schneelicht ausgesetzt wird. Diese abweichenden Angaben über die Zeit, in der die Erythropsie auftritt, muss den Verdacht erwecken, dass Vogt gar nicht die Erscheinungen der Erythropsie beobachtet, sondern nur das zu seinem Glas komplimentär gefärbte Nachbild. Eine Nachprüfung seiner Angaben können wir erst nächsten Winter vornehmen.

\section{Der Schutz des Auges gegen ultraviolette Strahlen.}

Aus den Arbeiten der erwähnten Autoren und aus unseren eigenen Untersuchungen ergibt sich:

1. dass unsere künstlichen Lichtquellen immer reicher an ultravioletten Strahlen geworden sind,

2. dass diese Strahlen dem Auge nachteilig sind,

3. dass die jetzt üblichen Glashüllen keinen genügen den Schutz vor diesen Strahlen bieten,

4. dass aber das Euphosglas solchen Schutz bietet, ohne die sichtbaren Strahlen wesentlich zu schwächen.

Bei diesem Sachverhalt verursacht die Beantwortung der Frage: Wie schützen wir unsere Augen vor der Einwirkung der ultravioletten Strahlen, keine Schwierigkeiten mehr. Wir müssen ein solches Euphosglas zwischen Auge und Lichtquelle einschalten. Dafür bieten sich zwei Wege. Wir können unsere Augen schützen durch Tragen 
einer Euphosglasbrille, oder wir können die Lichtquelle mit einer Euphosglashülle umgeben. Prof. Schulek hatte seinerzeit, da er bei seiner sehr eingehenden Untersuchung kein geeignetes Glas für diese Zwecke fand, eine Kammerbrille empfohlen. Diese Brille bestand aus einer schmalen, von zwei planparallelen Glasplatten begrenzten Kammer, in die eine Flüssigkeit gefüllt wurde, welche die ultravioletten Strahlen absorbierte. Diese Brille hat sich nicht eingeführt. Aus Euphosglas aber lassen sich Brillen herstellen genau wie die jetzt üblichen. Auf diesem Wege können wir unsern Augen leicht den nötigen Schutz bieten.

Wäre es aber nicht praktischer, den zweiten Weg vorzuziehen? Könnten wir den künstlichen Lichtquellen die ultravioletten Strahlen nicht ganz entziehen? Für den Sehakt werden die ultravioletten Strahlen nicht gebraucht. Haben sie sonst eine Bedeutung für den menschlichen Organismus? Wenn wir Pflanzen der Einwirkung gewisser Lichtarten aussetzen, so kommt das in der Entwicklung derselben zum Ausdruck. Die ultravioletten Strahlen spielen dabei wahrscheinlich eine ganz beachtliche Rolle. Auch für den menschlichen Organismus ist es sicher nicht gleichgültig, ob er der Einwirkung der ultravioletten Strahlen ganz entruickt ist oder nicht. Umgekehrt ist aber die Einwirkung von zu viel ultraviolettem Licht auf den Gesamtorganismus auch schädlich, das haben wir bei unseren Versuchen an uns selbst erfahren. Das Arbeiten in einem hellgestrichenen Raum bei einer Lampe, die ausserordentlich reich an ultravioletten Strahlen war, verursachte bei dem einen von uns (Schanz), der vorher nie mit solchem intensiven Licht gearbeitet hatte, nach mehrstündigem Aufenthalt in diesem Raum, obgleich direkte Bestrahlung nach Möglichkeit vermieden wurde, die erste Zeit ein solches Eingenommensein des Kopfes und ein solches Unbehagen, dass ein Weiterarbeiten auch unter anderer Beleuchtung für diesen Tag unmöglich war. 'Mit der Zeit trat aber eine Gewöhnung ein und jetzt fehlt eine derartige Empfindung bei gleicher Arbeit vollständig. Noch auffälliger war die Einwirkung der ultravioletten Strahlen auf das Allgemeinbefinden bei demselben Mitarbeiter, als er einem Vortrag mit Demonstration einer Quarzlampe beiwohnte. Er bekam ein unbehagliches Gefühl in der Magengrube und Beklemmung. Von dem Ingenieur eines Werkes, das sich mit der Herstellung elektrischer Lampen beschäftigt, wurde uns auch gesagt, dass dies eine bekannte Erscheinung sei und man deshalb die Arbeitsplätze so eingerichtet habe, dass die Magengrube der Arbeiter durch 
ein Blech vor der direkten Bestrahlung geschützt wird. Auf die Störungen an der Haut, die durch ultraviolette Strahlen entstehen und die zu therapeutischen $Z$ wecken jetzt auch häufig absichtlich erzeugt werden, wollen wir hier gar nicht näher eingehen.

Das Zuviel der ultravioletten Strahlen ist dem menschlichen Organismus schädlich und das Zuwenig wird ihm auch nachteilig sein. Wird dies von Einfluss sein müssen auf die Beantwortung der eben aufgeworfenen Frage? Die ultravioletten Strahlen, die der menschliche Organismus bedarf, hat er in der Natur in ausreichendem Masse, wir brauchen sie ihm nicht noch durch eine an ultravioletten Strahlen reiche Beleuchtung zuzuführen. Der Mensch, der nur in den Abendstunden bei künstlichem Licht arbeitet, kann seiner Lichtquelle sicher ohne Schaden alle ultravioletten Strahlen entziehen. Daher können wir ohne Bedenken unsere künstlichen Lichtquellen mit Glashüllen umgeben, die diese Strahlen vollständig absorbieren. Wir haben daher für alle Arten Lichtquellen Glashüllen aus Euphosglas herstellen lassen.

Dass unser Glas dem Auge einen vorzüglichen Schutz verleiht, lässt sich auch durch das Tierexperiment in sehr augenfälliger Weise zeigen. Bei unseren Versuchstieren haben wir die Lider auseinander genäht, um die Augen anhaltend zu belichten. Dabei sieht man, wie schon oben geschildert, die Fluorescenz der Linse. Schaltet man zwischen der Lichtquelle und dem Auge eine nur $1 / 2 \mathrm{~mm}$ dicke Glasplatte von Euphosglas ein, so schwindet die Fluorescenz sofort. Noch schöner wird der Versuch, wenn man die Strahlen erst durch ein blaues Uviolglas, das für ultraviolette Strahlen besonders gut durchlässig ist, gehen lässt. Die sichtbaren Strahlen werden bei diesem Glas alle bis auf die blauen und violetten Strahlen absorbiert. In dem dunkelblau beleuchteten Auge fluoresziert die Linse besonders schön. Bringt man jetzt ein Euphosglas von $1 / 2 \mathrm{~mm}$ Dicke zwischen Lichtquelle und Auge, so verschwindet wieder die Fluorescenz sofort. Aber noch interessanter wird der Versuch, wenn man das Euphosglas wieder entfernt. Die Fluorescenz tritt sofort wieder auf und gleichzeitig zucken die Lider des Tieres krampfhaft zusammen. Die ultravioletten Strahlen veranlassen einen äusserst beftigen Liderschlussreflex. Das Tier, das gleichmässig und ruhig in das Licht blickt, zuckt krampfhaft mit den Lidern zusammen, wenn die ultravioletten Strahlen, die das Euphosglas abgehalten hat, wieder sein Auge treffen. Einen besseren Beweis für die irritierende Wirkung der ultravioletten Strahlen auf 
Wie schützen wir unsere Augen v. d. Einwirk. d. ultraviol, Strahlen usw.

das Auge und die Schutzkraft des Fuphosglases kann es gar nicht geben.

Werfen wir jetzt nur noch einen kurzen Blick auf die verschiedenen Formen der künstlichen Beleuchtung. Die an ultravioletten Strahlen reichste Lichtquelle ist neben der elektrischen Bogenlampe das Quecksilberbogenlicht. Bei der Beleuchtung von Strassen und Plätzen scheint es nicht so bedenklich, teils weil die Lampen an hohen Masten angebracht sind, teils weil die Passanten verhältnismässig kurz den ultravioletten Strahlen ausgesetzt sind. Auch für Innenräume (Säle, Hallen u. dgl.) mag die Beleuchtung durch elektrische Bogenlampen noch einigermassen angängig sein, vorausgesetzt, dass die Lampen möglichst nahe an der Decke angebracht werden and die Belichtung des Raumes durch diffuse Reflexion erfolgt. Befremdlich ist es, dass die Industrie so wenig Wert auf eine derartige hygienische Beleuchtung legt. Die allermeisten Beleuchtungsanlagen sind nur nach dem Grundsatz hergestellt, für möglichst wenig Geld soll möglichst viel Licht erzeugt werden. $\mathrm{Ob}$ hierbei eine Belästigung, Schädigung oder sehr oft geradezu eine Vergewaltigung des Auges damit verbunden ist, wird ganz ausser acht gelassen. Man vergegenwärtige sich nur einmal die heutige Schaufensterbeleuchtung. Die direkte Beleuchtung des Arbeitsplatzes mit Bogenlicht ist sicher ganz unhygienisch und es hat sich dafür auch am meisten die Glühlampe eingebürgert, aber wohl auch mehr wegen ihrer Handlichkeit und Anpassungsfähigkeit an das Lichtbedurfnis als wegen ihrer augenhygienischen Vorteile. Sie steht häufig auf dem Arbeitsplatz direkt neben der Arbeit. Meist hält man eine besondere Umhüllung der Glühbirne für unnötig. Hygienische Gesichtspunkte werden bei dieser Art der Beleuchtung ganz vernachlässigt. Das Licht der Glühlampe ist sehr reich an ultravioletten Strahlen; zudem ist hier die Glashülle, die bei den andern künstlichen Lichtquellen durch ihre Dicke wenigstens einen, wenn auch nur geringen 'Teil der ultravioletten Strahlen abtängt, äusserst dünn. Die ausserordentlich grosse Flächenhelligkeit der Glühfäden wirkt direkt auf das Auge. Lange anhaltende Nachbilder belästigen den, der von seiner Arbeit aufblickt und dessen Blick dabei auf die Lampe fällt. Die Glühlampe ist deshalb schon als die giftigste Lichtquelle bezeichnet worden und dies nicht mit Unrecht!

Das Licht der Petroleum- und Gasglühlampen ist gegenüber den elektrischen Glühlampen etwas besser, dabei ist auch die Flächenhelligkeit des leuchtenden Körpers bedeutend geringer. Die Lichtquelle ist 
74 F. Schanz u. K. Stockhausen, Wie schützen wir unsere Augen v. d. Einwirk. usw.

meist durch mehrfache Glashüllen (Cylinder, Lampenglocke) umhüllt. Solche Lampen sind den elektrischen Glühlampen, obgleich sie nicht so handlich und bequem sind, in hygienischer Beziehung weit überlegen. Uns beiden war schon, ehe wir die Einwirkung des ultravioletten Lichtes auf das Auge kannten, eine gute Petroleumlampe ohne Glühstrumpf die angenehmste Arbeitslampe. Es liegt dies zweifellos daran, dass diese Lampe bei ausreichender Helligkeit ein Licht liefert, das nur wenig ultraviolette Strahlen enthält. Wir müssten diese Lampe auch nach unsern jetzigen Untersuchungen als die beste Arbeitslampe empfehlen, wenn wir kein Mittel gefunden hätten, das uns gestattet, den andern Lichtquellen die ultravioletten Strahlen zu entziehen.

Im Exphosglas besitzen wir ein solches Mittel. Das Glas ist etwas gelbgrünlich gefärbt. Der dadurch notwendigerweise entstehende Lichtverlust ist nicht zu vermeiden, er ist aber bei der erforderlichen Dicke des Glases nur so gering - im Durchschnitt $5 \%$-, dass er ausser acht gelassen werden kamn. Bei den zahlreichen Lichtquellen, die uns heute zur Beleuchtung zur Verfügung stehen, ist der Reichtum an ultravioletten Strahlen, wie wir gezeigt haben, je nach der Art der Lichtquelle verschieden. Auch die Dicke der gebräuchlichen Glasumbüllungen schwankt innerhalb gewisser Grenzen. Daraus ergibt sich für uns die Notwendigkeit, um eine unnötige Schwächung der sichtbaren Strahlen zu vermeiden, das Euphosglas genau den verschiedenen Lichtquellen anzupassen. Die Zusammensetzung des Glases muss sich daher je nach dem Zweck, dem es dienen soll, ändern, z. B. wird ein Glas, das für Glühlampenbirnen oder Gasglüblichtcylinder ausreichend ist, für eine Bogenlampe nicht genügen.

Auf Taf. XIV haben wir die Spektren von Lampen mit gewöhnlichen Glashüllen und von Lampen mit Euphosglashüllen wiedergegeben. Diese Tafel lässt erkennen, dass wir den Glasumbüllungen einer jeden Lichtquelle in ausreichendem Masse die Eigenschaft geben können, alle schädlichen ultravioletten Strahlen zu absorbieren. 
Lichtquellen: 0̈llampen.

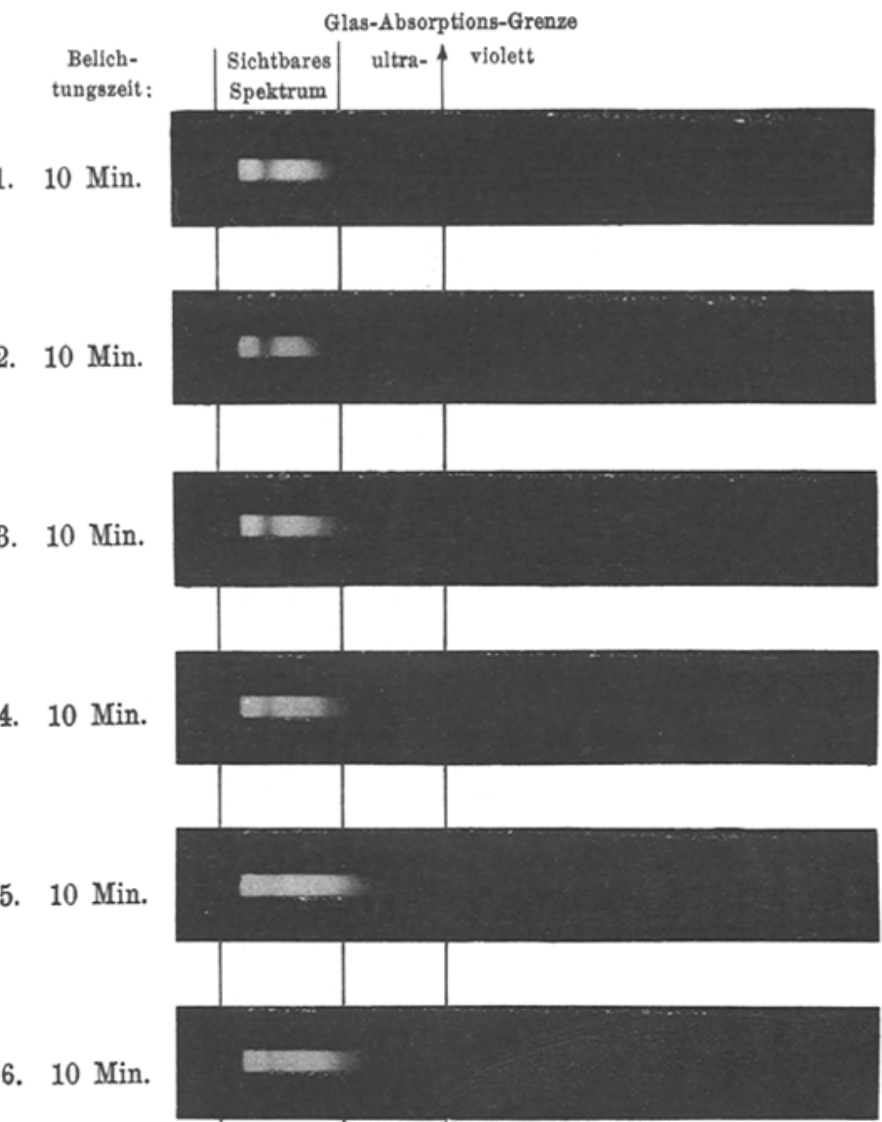

131. Römische 0livenöllampe 0,85 HK.

132. Walrat-Kerze $1,13 \mathrm{HK}$.

133. Stearin-Kerze $1,48 \mathrm{HK}$.

131a. Offene Rüböllampe $0,82 \mathrm{HK}$.

134. Moderateur-Lampe (Rüböl) mit Zylinder 11,4 HK.

129. May's ideal lamp (Petroleumlampe ohne Zylinder) $21,2 \mathrm{HK}$.

128. Petroleum-Sehnittbrenner 6,28 HK.

7. $10 \mathrm{Min}$.
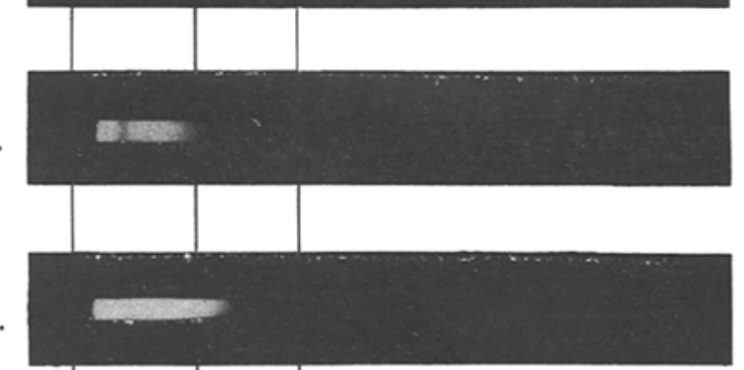

8. $10 \mathrm{Min}$.

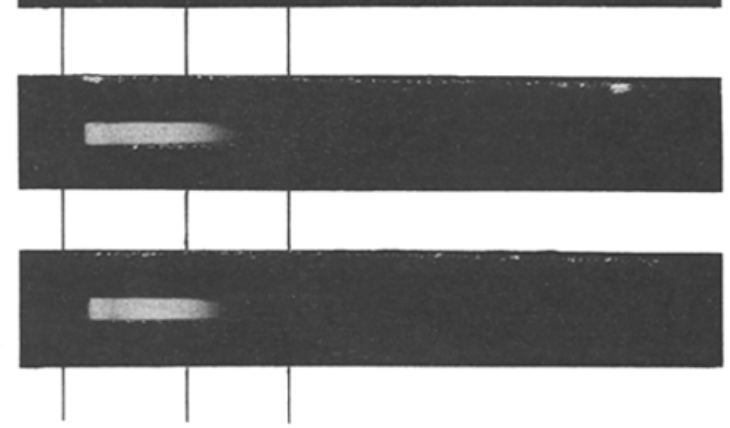

9. $10 \mathrm{Min}$

130a. Petroleum-Glühlampe 22,3 HK.

135. Spiritus-Glühlampe

10. 10 Min.

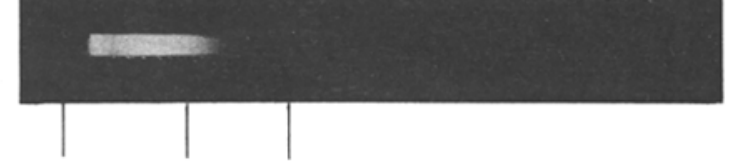

126. Petroleum-Rundbrenner $17,2 \mathrm{HK}$ 
v. Graefe's Archiv. Bd. LXIX.

\section{Lichtquellen: Gaslampen.}

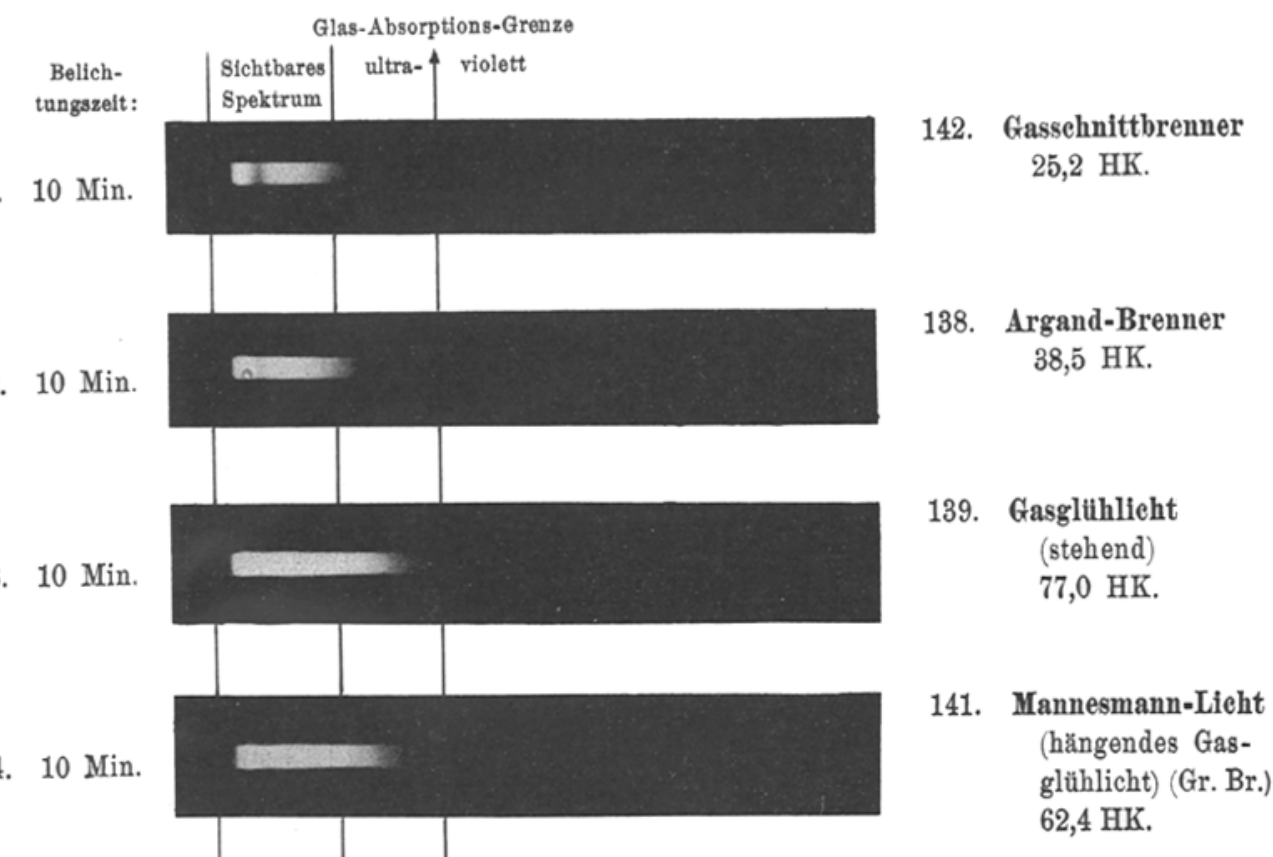

137. Kramer-Lieht

5. $10 \mathrm{Min}$.

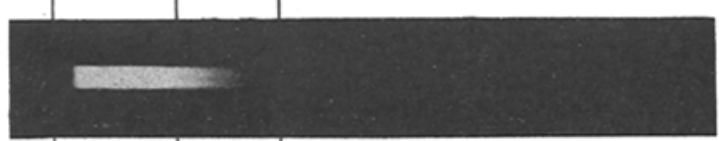

6. $10 \mathrm{Min}$.

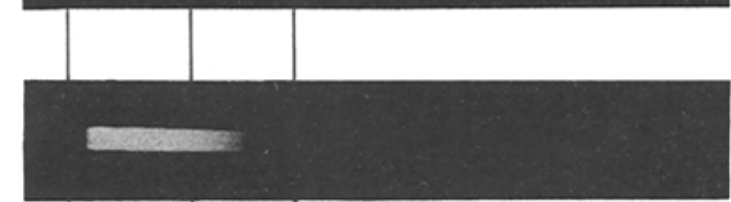

(Type U) (hängendes Gasglühlicht) $19,0 \mathrm{HK}$.

136a. Kramer-Lieht (Type K) (hăngendes Gasglühlicht) 55,4 HK.

140a. Ols0-Brenner No. 4 (stehendesGasglühlicht) $120 \mathrm{HK}$.

7. $10 \mathrm{Min}$.
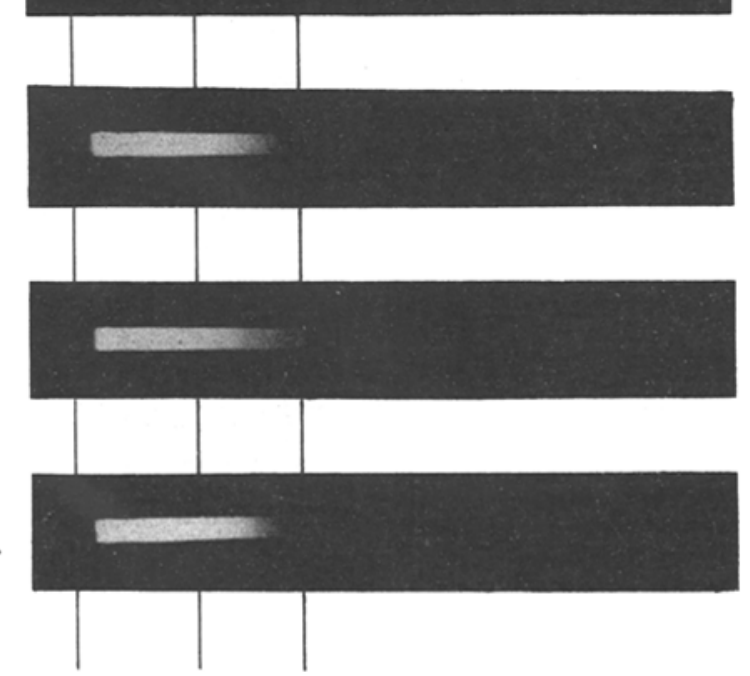

8. $10 \mathrm{Min}$.

145. Acetylen-Sehnittbrenner $27,5 \mathrm{HK}$.

146. Acetylen-Gluhlicht $32,6 \mathrm{HK}$.

9. $10 \mathrm{Min}$.

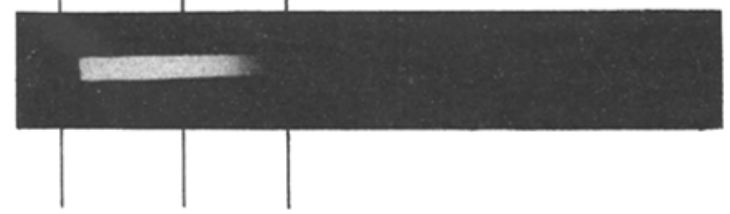

Verlag von Withelm Engelmann in Leipaig. 


\section{Lichtquellen: Elektrische Glühlampen.}

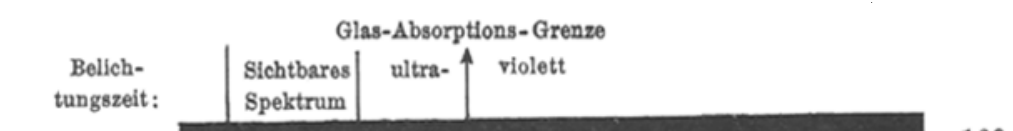

1. 20 Min.

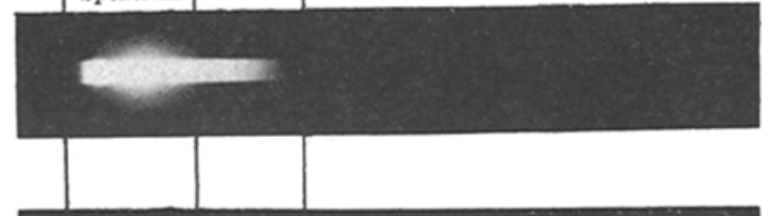

160. Kohlenfaden-Glithlampe 43,5 HK.

2. $10 \mathrm{Min}$.

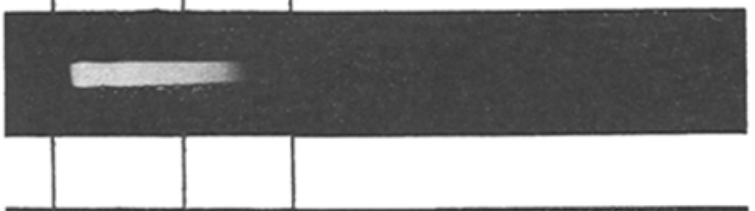

149. Tantallampe (klar) $41,8 \mathrm{HK}$.

3. $10 \mathrm{Min}$.

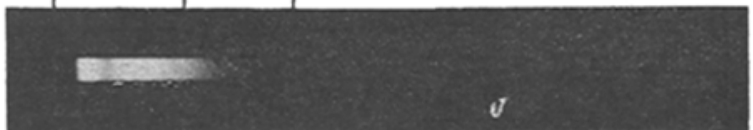

150. Tantallampe (matt) $26,7 \mathrm{HK}$.

4. $10 \mathrm{Min}$.
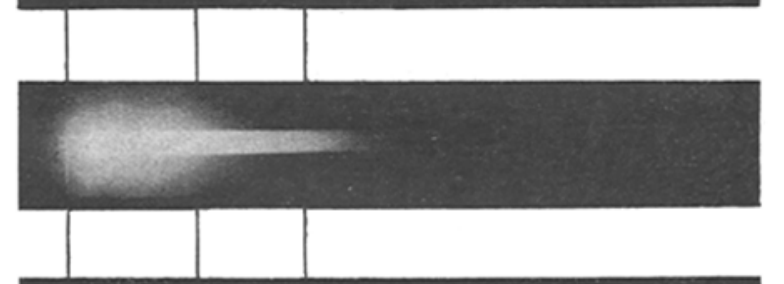

143. Nernstlampe ohne Glocke

5. $10 \mathrm{Min}$.

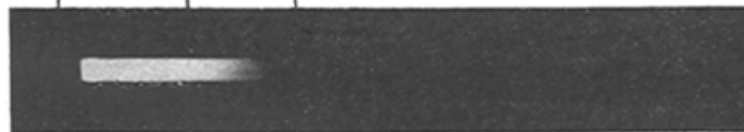

6. $10 \mathrm{Min}$.

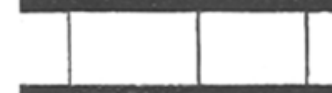

148. Nernstlampe mit Glocke 26,2 HK.

155. Zirkonlampe 45,5 HK.

7. 10 Min.

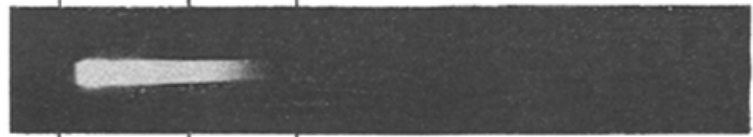

8. 10 Min.
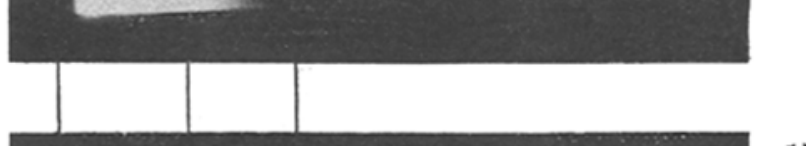

154. Osramlampe 39,8 HK.

151. Colloidlampe $37,1 \mathrm{HK}$.

152. Wolframlampe 63,7 HK. 


\title{
Lichtquellen: Elektrische Bogenlampen.
}

\author{
Glas-Absorptions-Grenze
}

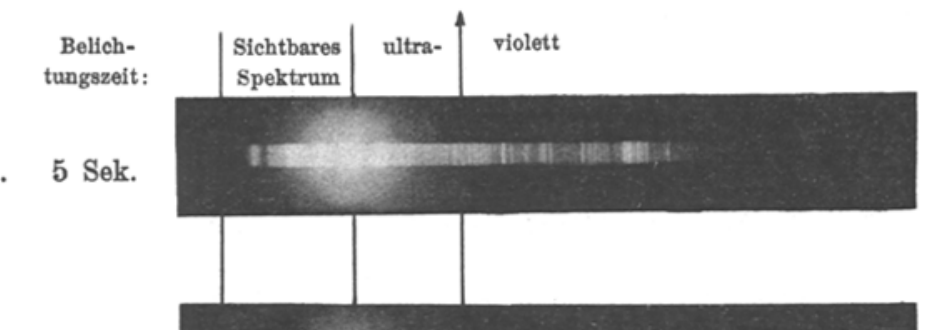

2. 5 Sek.

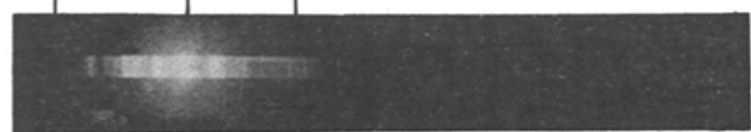

3. 10 Sek.

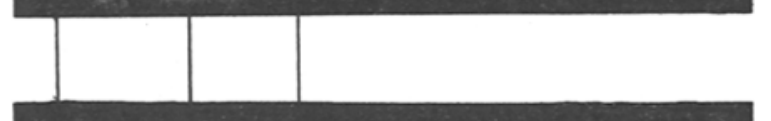

62. Offene Bogenlampe ohne Glocke 10 Amp.

423. Offene Bogenlampe mit Glocke 10 Amp.

13. Bogenlampe mit eingeschloss.Liehtbogen ohne Glocke 10 Amp.

117. Bogenlampe mit eingeschloss.Lichtbogen mit Glocke 10 Amp.

4. 5 Sek.
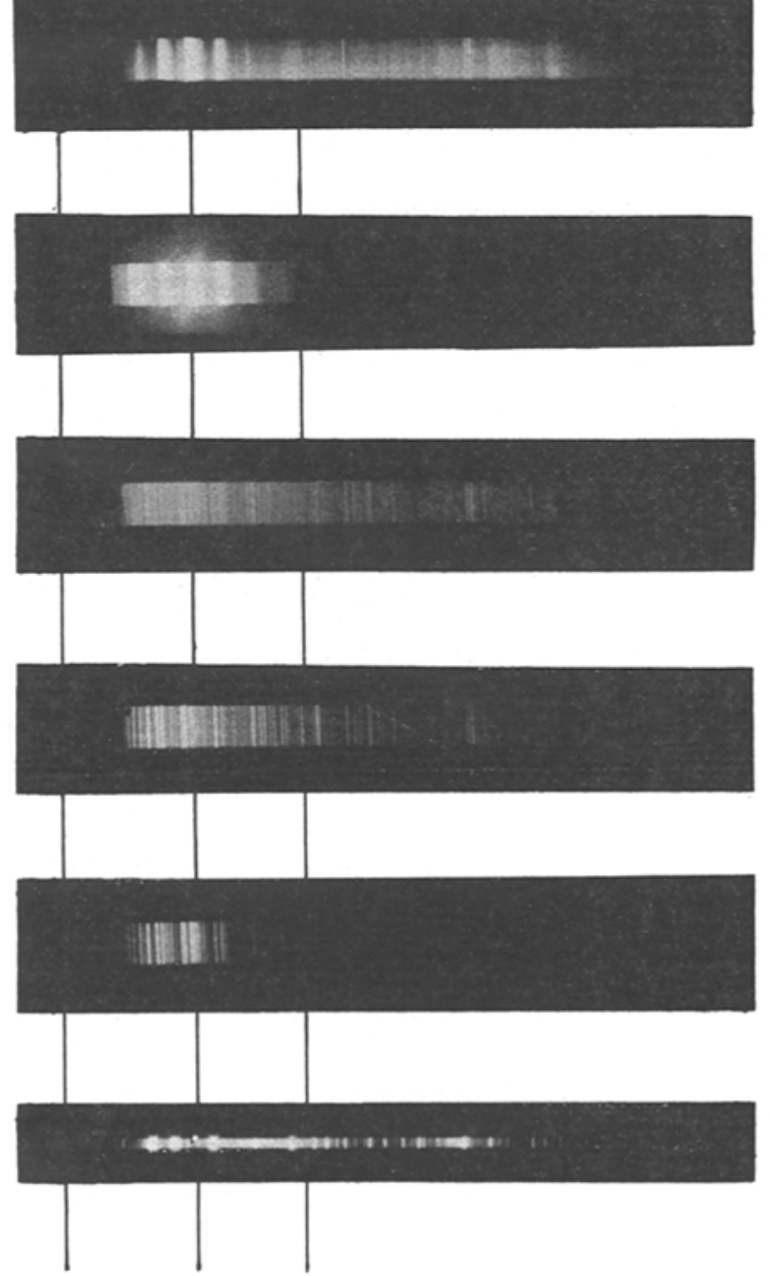

5. 5 Sek.

115. Intensiv-Flammenbogenlampe (weisse Effektkohlen) 10 Amp.

113. Intensiv-Flammenbogenlampe (gelbe Effektkohlen) 10 Amp.

114. Intensiv-Flammenbogenlampe mit Glocke (gelbe Effektkohlen) $10 \mathrm{Amp}$.

116. Quecksilberdampflampe mit Quarzumhüllung 


\section{Beleuchtungsgläser.}

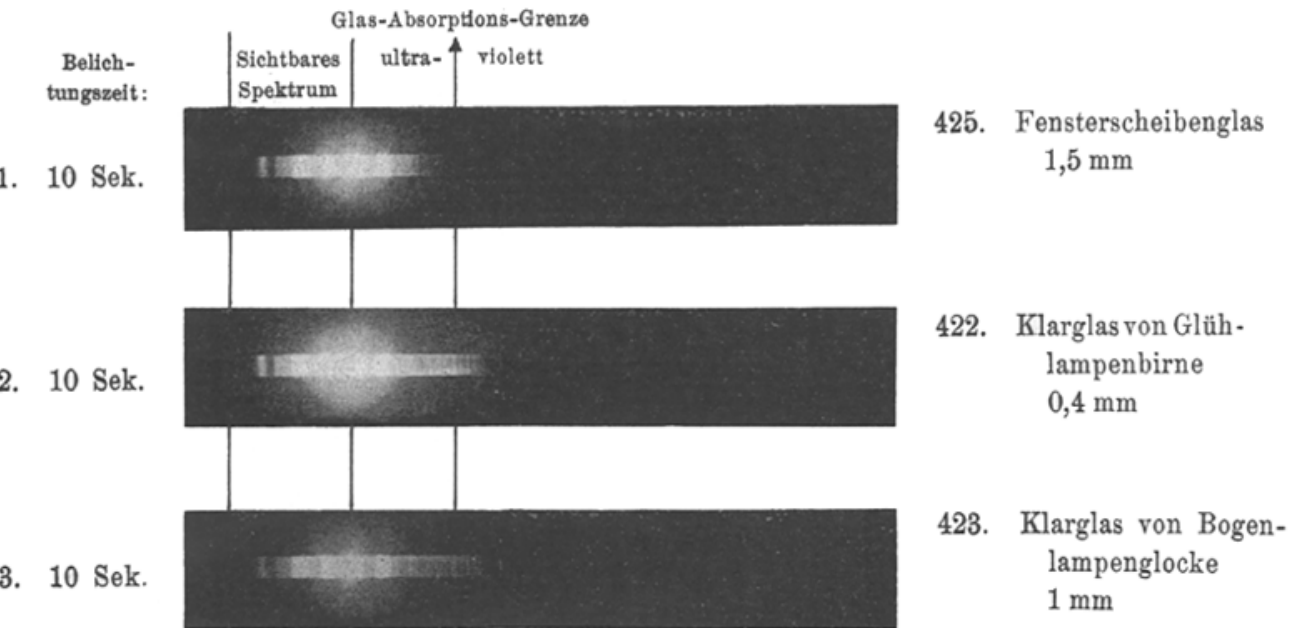

4. $10 \mathrm{Sek}$.

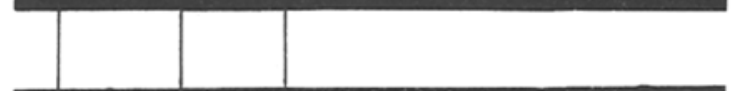

426. Kristallglasplatte

5. $10 \mathrm{Sek}$.
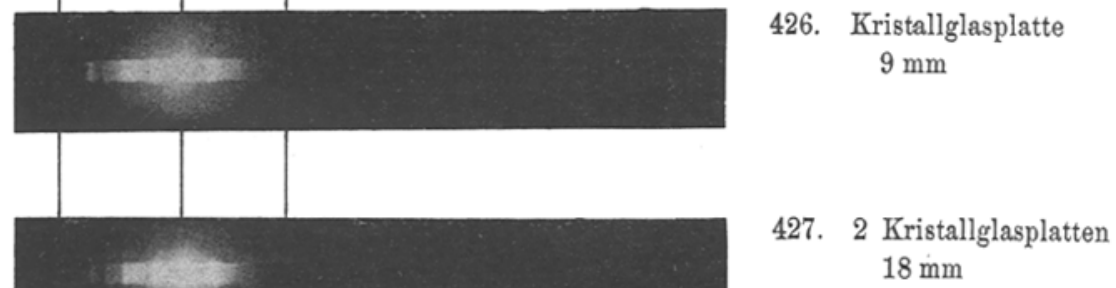

427. 2 Kristallglasplatten $18 \mathrm{~mm}$

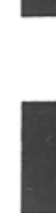
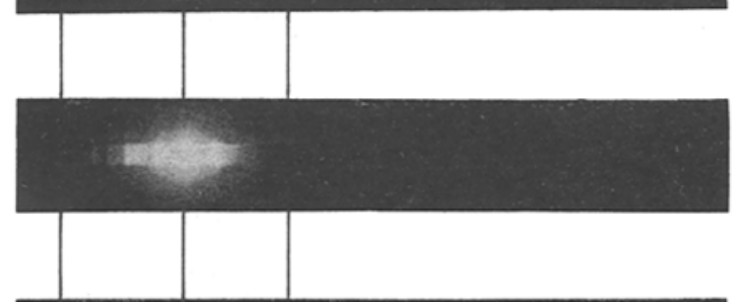

6. 10 Sek.

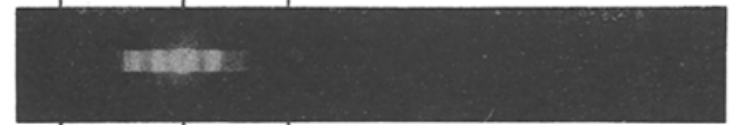

436. Petroleum-Zylinder

$$
1,5 \mathrm{~mm}
$$
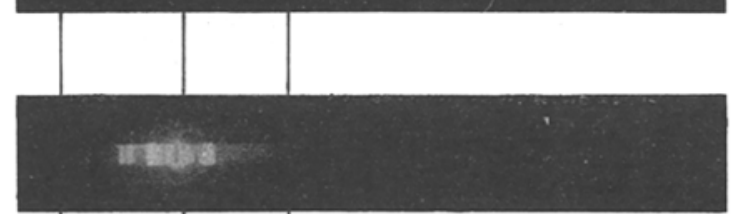

435. Gasglühlicht-Zylinder

7. 10 Sek.

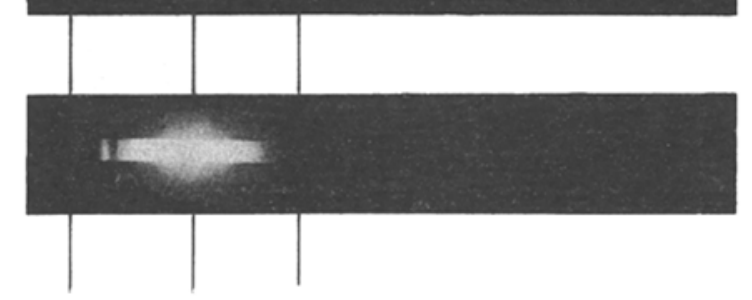
$1,0 \mathrm{~mm}$

466. Glimmer-Zylinder

8. 10 Sek.

$$
0,1 \mathrm{~mm}
$$

Verlag von Withelm Engelmann in Leipzig. 


\section{Beleuchtungsgläser.}

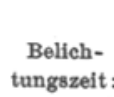

1. 10 Sek.

2. 10 Sek.

3. 1 Min.

4. $1 \mathrm{Min}$.

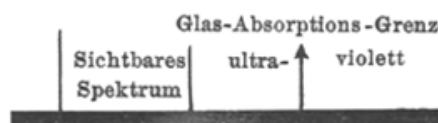

$$
5
$$

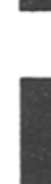

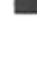
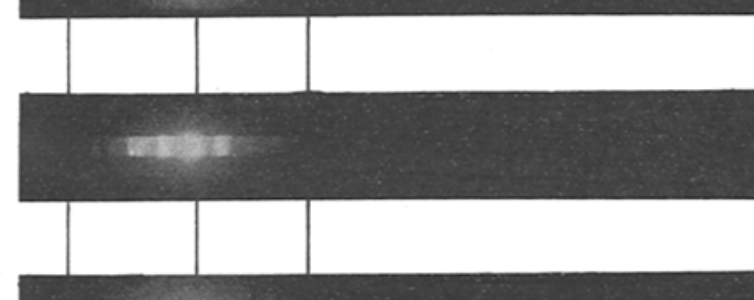

$$
\text { L }
$$

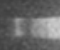

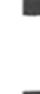

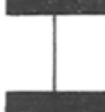

5. 1 Min.

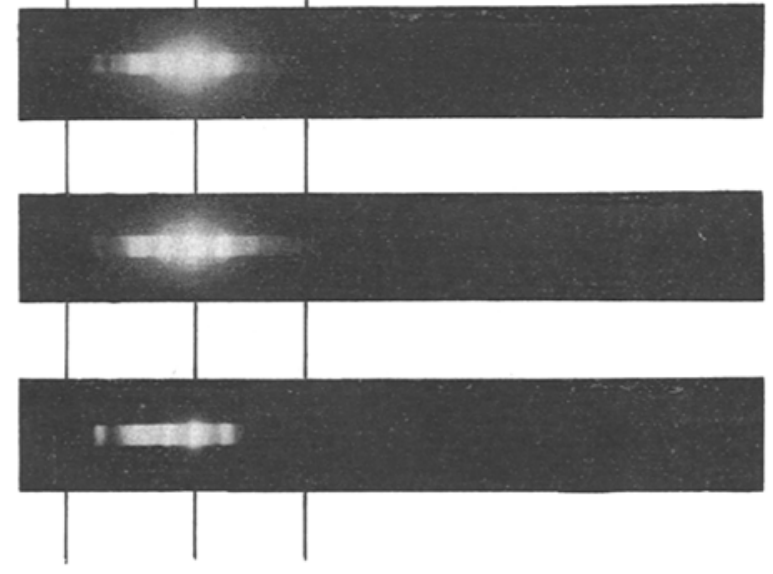

7. $4 \mathrm{St}$.
424. Gewöhnliches Brillenglas $2 \mathrm{~mm}$

454. Bleiglasbrille $4 \mathrm{~mm}$

631. Opalinglas

$$
1 \mathrm{~mm}
$$

625. Opalglas $2 \mathrm{~mm}$

626. Opalglas, dichter $2 \mathrm{~mm}$

627. Mattglas $1 \mathrm{~mm}$

665. Mllchglas, sehr dicht $1 \mathrm{~mm}$ 


\section{Brillengläser.}

\section{Blau.}

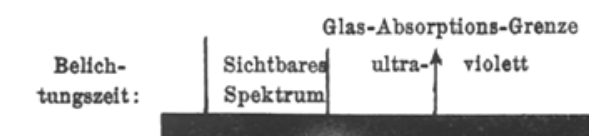

1. 10 Sek.

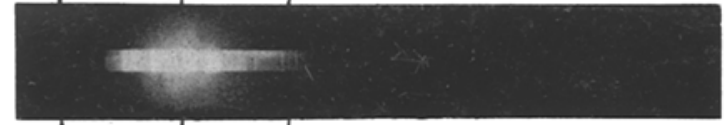

393. No. $21 \mathrm{~mm}$

2. 10 Sek.

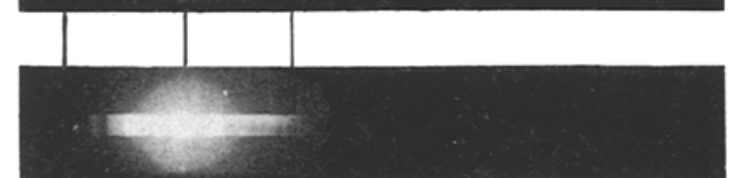

3. 10 Sek.

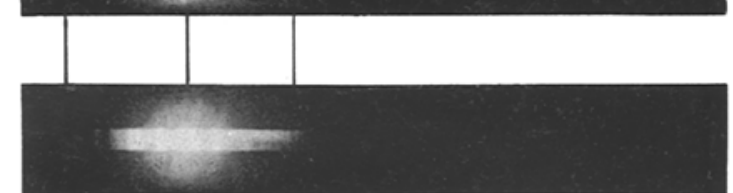

394. No. $4 \quad 1,5 \mathrm{~mm}$

4. 10 Sek.

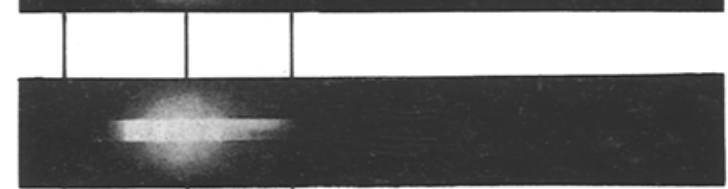

395. No. $6 \quad 2 \mathrm{~mm}$

396. No. $12 \quad 2 \mathrm{~mm}$

5. 20 Sek.

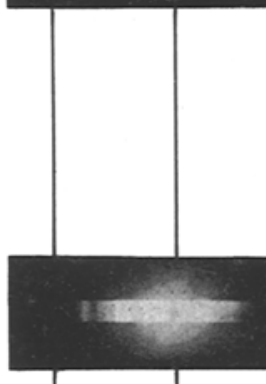

Rauchgrau.

6. 20 Sek.

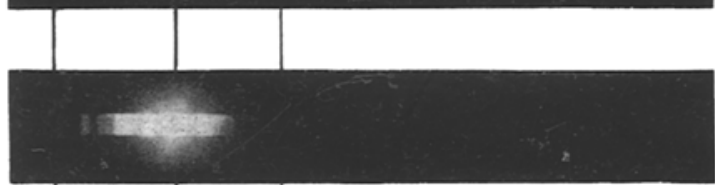

408. No. $2 \quad 1,5 \mathrm{~mm}$

7. 20 Sek.

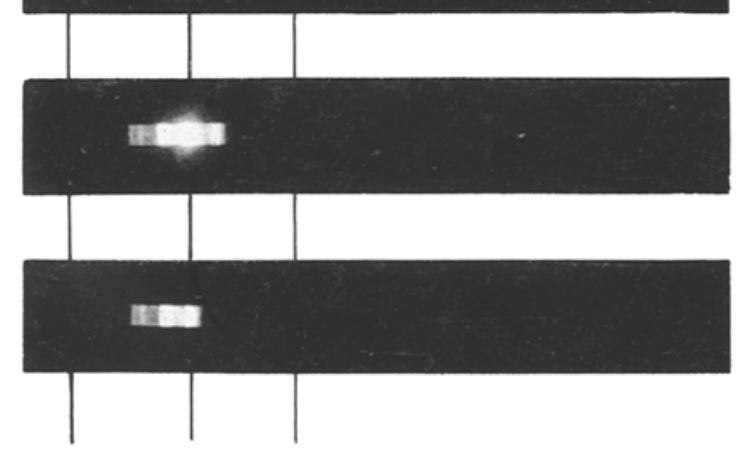

409. No. $4 \quad 1,5 \mathrm{~mm}$

8. 20 Sek.

411. No. $8 \quad 2 \mathrm{~mm}$

410. No. $72 \mathrm{~mm}$ 


\section{Brillengliser.}

Blen ardoise.

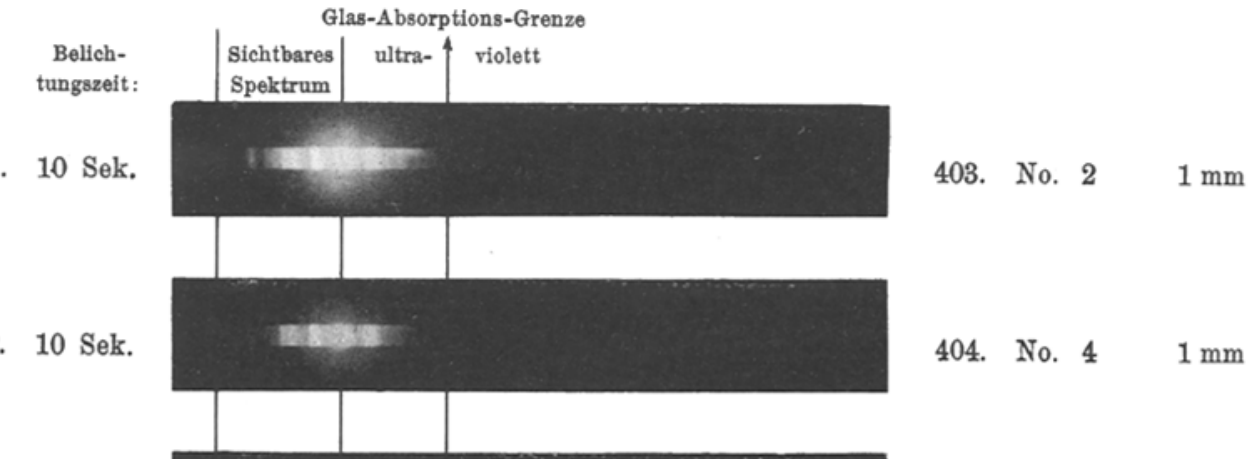

3. 10 Sek.

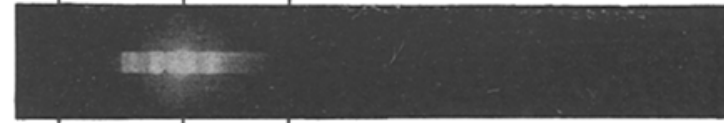

405. No. $51 \mathrm{~mm}$

4. $10 \mathrm{Sek}$.

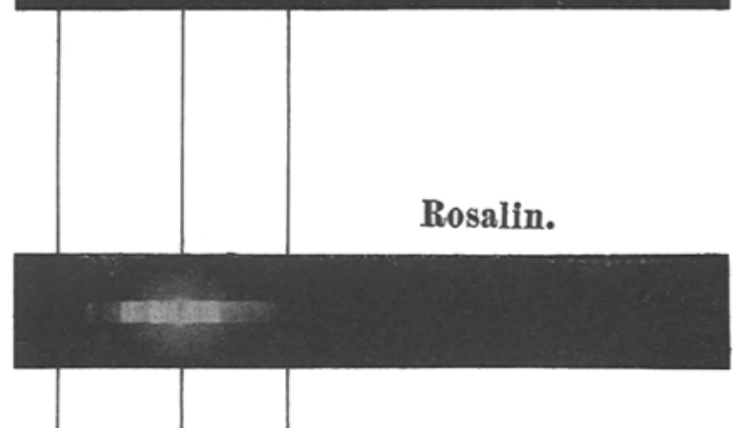

412. No. $12 \mathrm{~mm}$

5. 10 Sek.

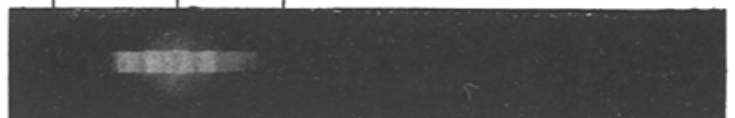

413. No. $22 \mathrm{~mm}$

6. 10 Sek.

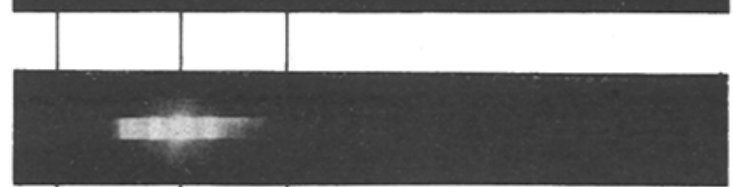

414. No. $4 \quad 2 \mathrm{~mm}$

7. 10 Sek.

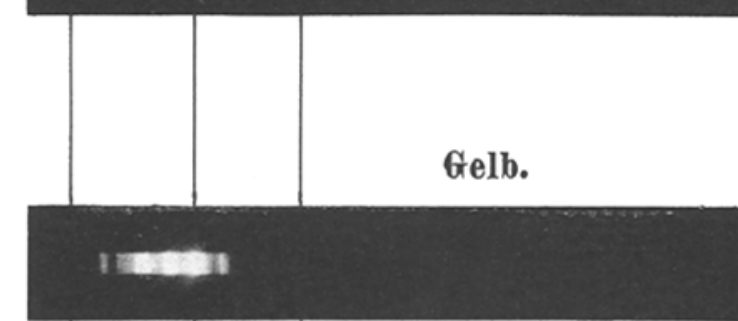

406. No. $12 \mathrm{~mm}$

8. 10 Sek.

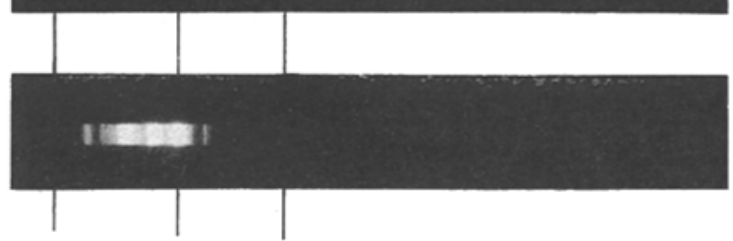

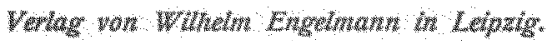

407. No. $22 \mathrm{~mm}$ 


\section{Fieuzal-, Enixanthos, Lichtilter- and Euphos-9litser.}

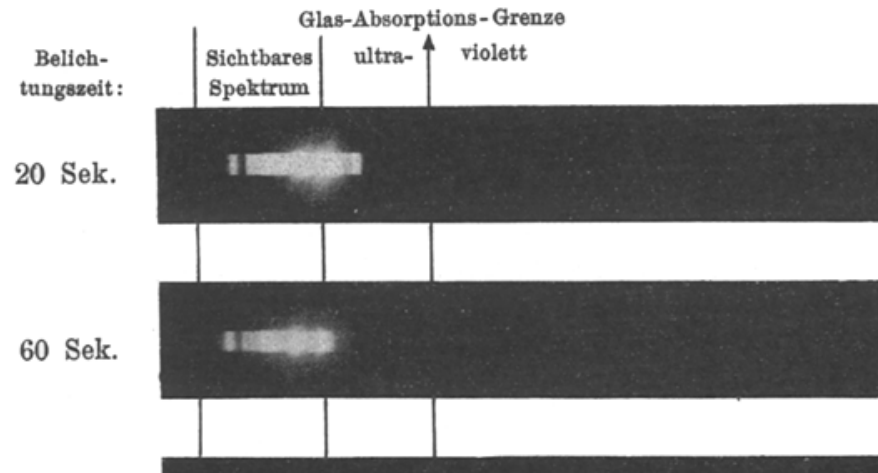

3. 3 Min.

4. 40 Sek.

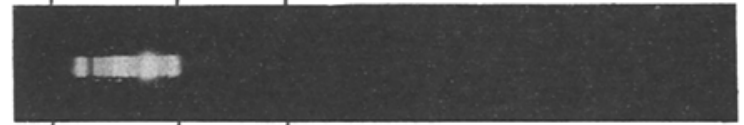

5. 5 Min.

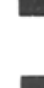

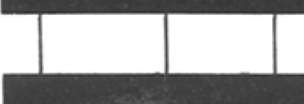

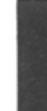

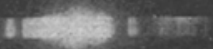

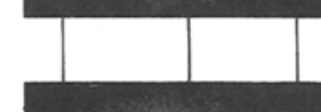

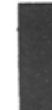

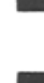

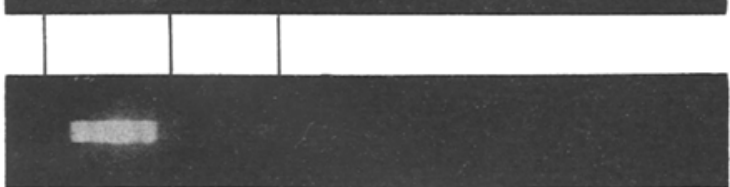

6. 5 Min.

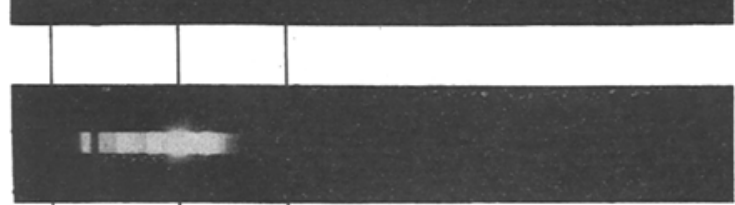

7. 20 Sek.

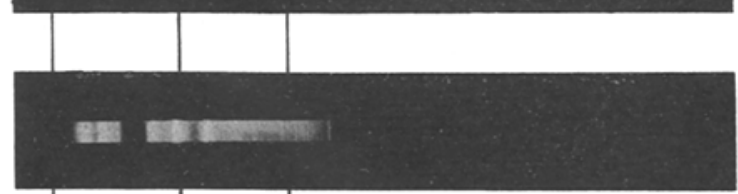

8. 20 Sek.

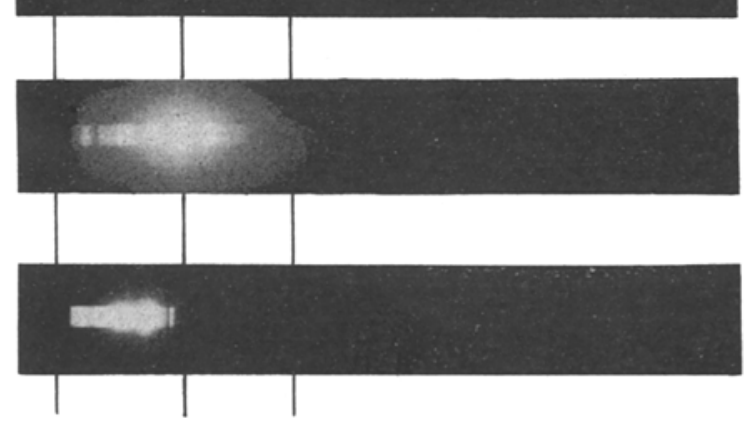

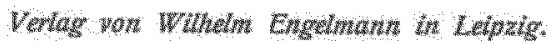

10. 20 Sek.
389. Fieuzalglas No. 1 $1 \mathrm{~mm}$

636. Fieuzalglas No. 2 $2 \mathrm{~mm}$

637. Fieuzalglas No. 3 $2 \mathrm{~mm}$

638. Enixanthosglas No. 1 $2 \mathrm{~mm}$

640. Enixanthosglas No. 3 $2 \mathrm{~mm}$

81. Gelbes Jagdglas $2 \mathrm{~mm}$

388. Photograph. Gelbfilter hellgrüngelb $0,1 \mathrm{~mm}$

651. Photograph. Gelbfilter dunkelgelb $2 \mathrm{~mm}$

679. Euphosglas No. 72 $0,8 \mathrm{~mm}$ 
Giehtquellen nit Euphosglas.

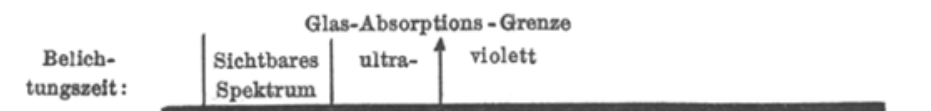

1. 5 Sek.

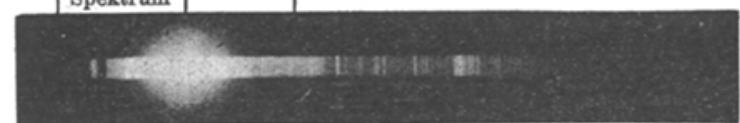

62. Offene Bogenlampe ohne Glocke 10 Amp.

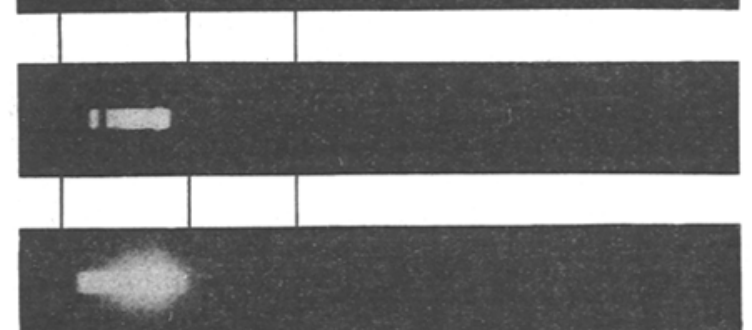

2. 5 Sek.

679. Dieselbe mit Euphosglasglocke $0,8 \mathrm{~mm}$ No. 72

3. 5 Min.

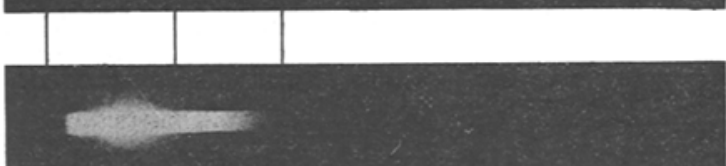

680. Dieselbe mit Euphosglasglocke $0,8 \mathrm{~mm}$ No. 72

160. Kohlenfadenglühlampe mit Klarglasbirne $0,5 \mathrm{~mm}$

4. $20 \mathrm{Min}$.

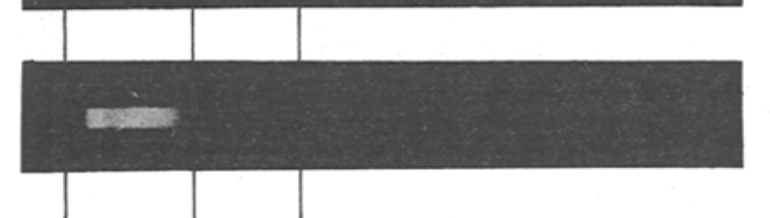

5. $20 \mathrm{Min}$.

575. Dieselbe mit Euphosglasbirne $0,5 \mathrm{~mm} \quad$ No. 71

154. Osramglühlampe mit Klarglasbirne $0,5 \mathrm{~mm}$

6. $10 \mathrm{Min}$.

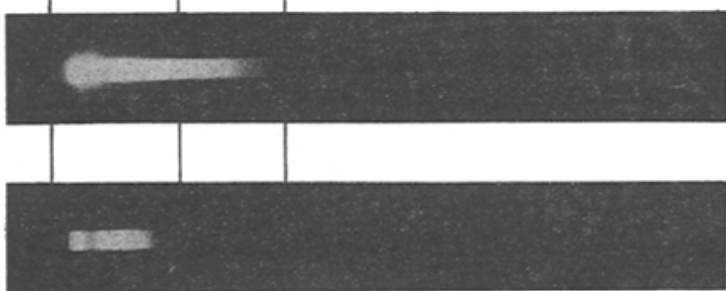

7. $10 \mathrm{Min}$.

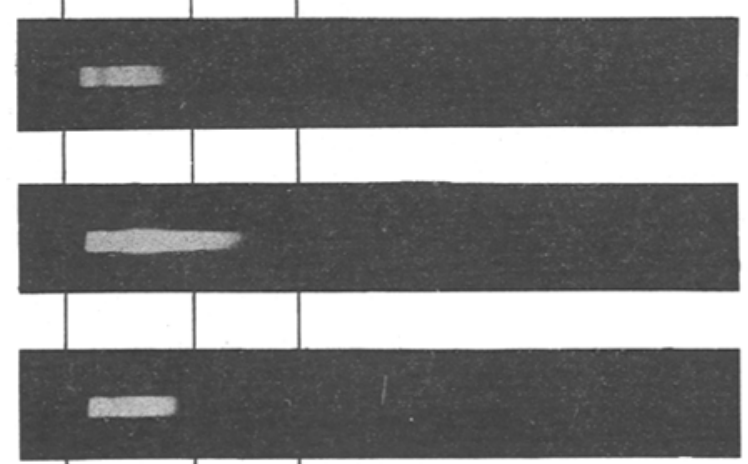

558. Osramglühlampe mit Euphosglasbirne $0,5 \mathrm{~mm}$ No. 71

685. Petroleum - Rundbrenner mit klarem Zylinder

686. Derselbe mit EuphosZylinder

9. $40 \mathrm{Min}$.

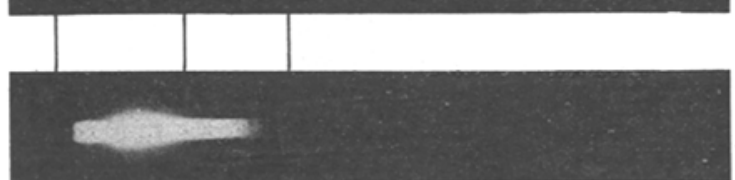

687. Gasglühlicht mit klarem Zylinder

10. $40 \mathrm{Min}$.

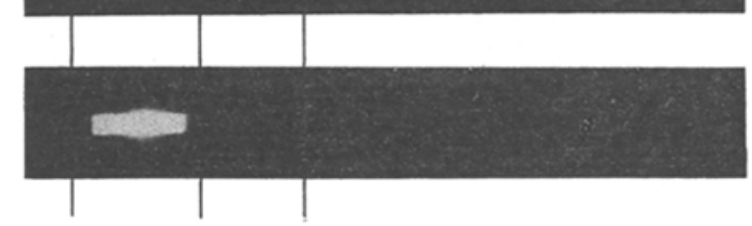

688. Dasselbe mit Euphos-

Zylinder

11. $40 \mathrm{Min}$. 